\title{
Output Feedback and Single-Phase Sliding Mode Control for Complex Interconnected Systems
}

\author{
Yao-Wen Tsai ${ }^{1}$ and Van Van Huynh ${ }^{2}$ \\ ${ }^{1}$ Department of Mechanical and Automation Engineering, Da-Yeh University, No. 168, University Road, Changhua 51591, Taiwan \\ ${ }^{2}$ Faculty of Electrical and Electronics Engineering, Ton Duc Thang University, 19 Nguyen Huu Tho Street, 7th District, \\ Ho Chi Minh City, Vietnam \\ Correspondence should be addressed to Yao-Wen Tsai; ywtsai.tw@gmail.com
}

Received 29 August 2014; Accepted 25 December 2014

Academic Editor: Xudong Zhao

Copyright (C) 2015 Y.-W. Tsai and V. V. Huynh. This is an open access article distributed under the Creative Commons Attribution License, which permits unrestricted use, distribution, and reproduction in any medium, provided the original work is properly cited.

\begin{abstract}
This paper generalized a new sliding mode control (SMC) without reaching phase to solve two important problems in the stability of complex interconnected systems: (1) a decentralized controller that uses only output variables directly and (2) the stability of complex interconnected systems ensured for all time. A new sliding surface is firstly designed to construct a single-phase SMC in which the desired motion is determined from the initial time instant. A new lemma is secondly established for the controller design using only output variables. The proposed single-phase SMC and the decentralized output feedback controller ensure the robust stability of complex interconnected systems from the beginning to the end. One of the key features of the single phase SMC scheme is that reaching time, which is required in most of the existing two phases of SMC approaches to stabilize the interconnected systems, is removed. Finally, a numerical example is used to demonstrate the efficacy of the method.
\end{abstract}

\section{Introduction}

The theory of sliding mode control (SMC) is known to be an effective robust control technique and has been successfully applied to a wide variety of practical engineering systems such as robot manipulators, aircrafts, underwater vehicles, spacecrafts, flexible space structures, electrical motors, power systems, and automotive engines [1]. The main advantages of SMC are fast response and strong robustness with respect to uncertainties and external disturbances [2-4]. Generally speaking, the traditional SMC design can be divided into two phases: the reaching phase and the sliding phase. Firstly, in the reaching phase, the feature of SMC is to use a switching control law to drive system state trajectories onto a switching surface and remain on it thereafter. Secondly, in the sliding phase, the essence of SMC is to keep the state trajectories moving along the surface towards the origin with desired performance $[5,6]$.

Unfortunately, the applications of two phases SMC for the stability of complex interconnected system have some drawbacks. Firstly, the system stability is not ensured for all time because the motion equation in sliding mode is determined after the system state hits the sliding surface $[6$, 7]. Secondly, the performance of system in the reaching phase is unknown and, subsequently, global performance may be seriously degraded $[6,7]$. In addition, the state variables of complex interconnected system are not always accessible in many practical systems. Therefore, for complex interconnected systems, there are some important tasks should be solved: (1) the creation of a decentralized controller that uses only output variables directly; (2) guaranteed stability of complex interconnected systems for all time.

In order to solve the above problems, first we develop a new SMC such that the reaching time is equal to zero and the desired motion is determined from the beginning time. Second, appropriate LMI stability conditions by the Lyapunov method are derived to guarantee the stability of the system. Third, a new lemma is established for controller design using only output variables directly. Consequently, the stability of complex interconnected systems driven by singlephase SMC law can be ensured throughout an entire response of the system starting from the initial time instance. Before 
demonstrating the advantages of the application of singlephase SMC to complex interconnected systems, one wants to point out some previous results about the stability analysis of uncertain systems.

The design of SMC without reaching phase can be found in $[1,7-12]$. The authors of $[1,8]$ have presented a new method to design an integral sliding mode control law. This nice feature of the integral SMC law compensates the generally slower and more oscillatory transient [1]. In order to reduce disturbance, Rubagotti et al. [9] developed an integral sliding mode controller with state-dependent drift and input matrix. More recently, the researchers in [10] proposed a universal fuzzy integral SMC for mismatched uncertain systems, which does not require that all local linear systems share a common input matrix. The authors of [11] developed an integral SMC for handling a larger class of mismatched uncertainties. In [12], a new approach was proposed for approximating the system states and disturbance vectors using observer-based integral SMC. In addition, the stability of the sliding mode in terms of linear matrix inequalities (LMI) has some benefits over conventional approach methods, where LMI problems can be easily determined and efficiently solved using the LMI Toolbox in MATLAB software. As a result, the robustness of the integral SMC via the LMI technique is guaranteed throughout its entire trajectories starting from the initial time.

Thus, the approaches in $[1,7-12]$ cannot be directly applied to complex interconnected systems in which only output information is available. In the limited available literature, the associated decentralized output feedback results are few. In particular, when the mismatched uncertainties are included, only a few results are available [13-19]. Earlier works on decentralized SMC were mainly focused on interconnected systems or nonlinear systems with the matching condition [20-23]. A decentralized model reference adaptive control scheme is proposed in [24] in which the interconnections considered are linear and matched. In [25], sufficient stability conditions were derived for the switched interconnected time-delayed systems. The authors in [13] proposed a decentralized sliding mode controller for a class of mismatched uncertain interconnected systems by using two sets of switching surfaces where the exogenous disturbance was not mentioned. In $[14,15]$, a decentralized SMC scheme was proposed for a class of interconnected time-delayed systems with dead-zone input. In [16], a multiple-sliding surface control scheme is presented for a class of multiinput perturbed systems. In [17], a decentralized dynamic output feedback sliding mode controller is designed for mismatched uncertain interconnected systems. In [18], a global decentralised static output feedback SMC control scheme is proposed for interconnected time-delayed systems where the interconnection terms are functions of the output. In [19], a state observer-based sliding mode control is designed for a class of switched systems in which the system states are unmeasurable. The above works obtained important results related to handling the effects of interconnections and disturbances of interconnected systems using SMC theory. As a result, the stability of interconnected systems was assured under certain conditions.
However, it is worth to point out that there are some limitations in the existing design methods of SMC in application for the stability of interconnected systems. First, the approaches proposed in [20-25] could not be applied for mismatched uncertain interconnected systems. Second, the control schemes given in [13-24] are based on the traditional SMC method which only yields the desired motion after sliding motion has occurred. Therefore, the global performance may be seriously degraded. Hence, it is necessary to develop a new SMC without reaching phase to stabilize complex interconnected systems for all time.

This study therefore developed a new single-phase SMC for robust stability of a class of complex interconnected systems from beginning to end. First, a new sliding surface is designed to construct the single-phase SMC which the desired motion is determined from the initial time instant. Second, appropriate LMI stability conditions by the Lyapunov method are derived to guarantee the stability of the system. Third, a new lemma is established for controller design using only output variables. Fourth, a decentralized output feedback controller is designed to force the system states to stay on the sliding surface for all time. Unlike the existing related works such as $[1,7-12]$, this method can be directly applied for complex interconnected systems in which only output information is available. In contrast to the other SMC approaches given in [13-24], this approach guarantees the stability of complex interconnected systems for all time. In addition, the complex interconnected systems investigated in this study include exogenous disturbance, mismatched parameter uncertainties in the state matrix, and mismatched interconnections. Therefore, we consider a more general structure than [13-25]. To summarize, the main contributions of this paper are as follows.

(i) Design of a new sliding surface to construct a singlephase SMC such that the desired motion is determined from the initial time instant.

(ii) Derivation of appropriate LMI stability conditions by the Lyapunov method to guarantee the stability of the system.

(iii) Establishment of a lemma for controller design using only output variables.

(iv) Development of a new approach (single-phase SMC and decentralized output feedback controller) guarantees that sliding mode exists from the initial time instant and the closed loop of the complex interconnected systems in sliding mode is asymptotically stable.

Notation. The notation used throughout this paper is fairly standard. $X^{T}$ denotes the transpose of matrix $X . I_{n \times m}$ and $0_{n \times m}$ are used to denote the $n \times m$ identity matrix and the $n \times m$ zero matrix, respectively. The subscripts $n$ and $n \times m$ are omitted where the dimension is irrelevant or can be determined from the context. $\|x\|$ stands for the Euclidean norm of vector $x$ and $\|A\|$ stands for the matrix induced norm of the matrix $A$. The expression $A>0$ means that $A$ is symmetric positive definite. $R^{n}$ denotes the 
$n$-dimensional Euclidean space. For the sake of simplicity, sometimes function $x_{i}(t)$ is denoted by $x_{i}$.

\section{Problem Formulation and Preliminaries}

In this paper, we consider a class of complex interconnected systems with exogenous disturbance and mismatched uncertainties of each isolated subsystem and interconnection. The system is decomposed into $L$ subsystems and the state space representation of each subsystem is described as follows:

$$
\begin{aligned}
\dot{x}_{i}= & \left(A_{i}+\Delta A_{i}\right) x_{i}+B_{i}\left(u_{i}+\xi_{i}\left(x_{i}, t\right)\right) \\
& +\sum_{\substack{j=1 \\
j \neq i}}^{L}\left(H_{i j}+\Delta H_{i j}\right) x_{j}, \\
y_{i}= & C_{i} x_{i},
\end{aligned}
$$

where $x_{i} \in R^{n_{i}}, u_{i} \in R^{m_{i}}$, and $y_{i} \in R^{p_{i}}$ with $m_{i}<p_{i}<n_{i}$ are the state variables, inputs, and outputs of the $i$ th subsystem, respectively. The triples $\left(A_{i}, B_{i}, C_{i}\right)$ and $H_{i j}$ represent known constant matrices of appropriate dimensions. The matrices $\Delta A_{i} x_{i}$ and $\Delta H_{i j} x_{j}$ represent the mismatched parameter uncertainty in the state matrix in each isolated subsystems and mismatched interconnections, respectively. The matrix $B_{i} \xi_{i}\left(x_{i}, t\right)$ is disturbance input. In this paper, only the output variables $y_{i}$ are assumed to be known.

In order to modify the existing two phases SMC, we denote the sliding surface by $\sigma_{i}\left(x_{i}(t), t\right)=0, i=1,2, \ldots, L$, where the single-phase sliding function is given as

$$
\sigma_{i}\left(x_{i}(t), t\right)=\bar{\sigma}_{i}\left(y_{i}(t), t\right)-\bar{\sigma}_{i}\left(y_{i}(0), 0\right) \exp \left(-\beta_{i} t\right)
$$

with constant $\beta_{i}>0$. The function $\bar{\sigma}_{i}\left(y_{i}(t), t\right)$ is defined later. The sliding mode is defined by $\sigma_{i}\left(x_{i}(t), t\right)=0$ and $\dot{\sigma}_{i}\left(x_{i}(t), t\right)=0$. From (2), one can see that there are only output variables used and the system states are in the sliding mode from the initial time; $\sigma_{i}\left(x_{i}(0), 0\right)=0$. Therefore, this is to say that the SMC is single-phase (without reaching phase). This can be formally defined as follows.

Definition 1. A sliding mode control is said to be a singlephase SMC, if and only if the following two conditions are satisfied:

(1) the reaching time is equal to zero; $\sigma_{i}\left(x_{i}(0), 0\right)=0$;

(2) the order of the motion equation in sliding mode is equal to the order of the original system.

Remark 2. The concept of single-phase sliding mode control focusses on the robustness of the motion in the entire state space. The order of the motion equation in sliding mode is equal to the dimension of the state space. Therefore, the robustness of complex interconnected systems can be assured throughout an entire response of the system starting from the initial time instance.

In order to apply the concept of single-phase SMC for the system (1), we assume the following to be valid.
Assumption 3. The mismatched parameter uncertainties in the state matrix of each isolated subsystem are satisfied as $\Delta A_{i}=D_{i} F_{i}\left(x_{i}, t\right) E_{i}$ where $F_{i}\left(x_{i}, t\right)$ is unknown but bounded as $\left\|F_{i}\left(x_{i}, t\right)\right\| \leq 1$ and $D_{i}, E_{i}$ are known matrices of appropriate dimensions.

Assumption 4. The matrices $B_{i}$ and $C_{i}$ are full rank and $\operatorname{rank}\left(C_{i} B_{i}\right)=m_{i}$.

From [18], Assumption 4 implies that there exists a nonsingular linear coordinate transformation $\widetilde{z}_{i}=\widetilde{T}_{i} x_{i}$ such that the triple $\left(A_{i}, B_{i}, C_{i}\right)$ with respect to the new coordinates has the structure

$$
\widetilde{A}_{i}=\left[\begin{array}{cc}
\widetilde{A}_{i 1} & \widetilde{A}_{i 2} \\
\widetilde{A}_{i 3} & \widetilde{A}_{i 4}
\end{array}\right], \quad \widetilde{B}_{i}=\left[\begin{array}{c}
0 \\
\widetilde{B}_{i 2}
\end{array}\right], \quad \widetilde{C}_{i}=\left[\begin{array}{ll}
0 & \widetilde{C}_{i 2}
\end{array}\right],
$$

where $\widetilde{A}_{i 1} \in R^{\left(n_{i}-m_{i}\right) \times\left(n_{i}-m_{i}\right)}, \widetilde{B}_{i 2} \in R^{m_{i} \times m_{i}}$ are nonsingular and $\widetilde{C}_{i 2} \in R^{p_{i} \times p_{i}}$ is orthogonal.

Assumption 5. The triple $\left(\widetilde{A}_{i 1}, \widetilde{A}_{i 2}, \Xi_{i}\right)$ is output feedback

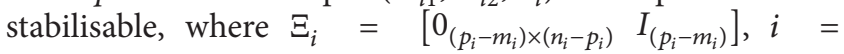
$1,2,3, \ldots, L$.

From [18], Assumption 5 implies that there exist matrices $\widetilde{K}_{i}$ such that the matrices $A_{i 1}=\widetilde{A}_{i 1}-\widetilde{A}_{i 2} \widetilde{K}_{i} \Xi_{i}$ are stable.

Assumption 6. There exist known nonnegative constants $c_{i}$ and $b_{i}$ such that $\left\|\xi_{i}\left(x_{i}, t\right)\right\| \leq c_{i}+b_{i}\left\|x_{i}(t)\right\|$.

Assumption 7. The mismatched interconnections are given as $\Delta H_{i j}=M_{i j} F_{i j}\left(x_{j}, t\right) N_{i j}$, where $F_{i j}\left(x_{j}, t\right)$ is unknown but bounded $\left\|F_{i j}\left(x_{j}, t\right)\right\| \leq 1$, and $M_{i j}, N_{i j}$ are known matrices of appropriate dimensions.

Remark 8. Assumptions 4 and 5 have been utilized in [18]. The assumption of the norm boundedness of $F_{i}\left(x_{i}, t\right)$ and $\xi_{i}\left(x_{i}, t\right)$ can be found in $[19,26,27]$.

\section{Single-Phase Sliding Mode Control for Complex Interconnected Systems}

In this section, we develop a single-phase SMC to stabilize the complex interconnected system (1) for all time. There are four steps involved in the design of our single-phase SMC using only output variables. In the first step, a proper sliding surface is designed to construct the single-phase SMC such that the desired motion is determined from the initial time instant. In the second step, sufficient conditions in terms of LMI are derived for the existence of a sliding surface guaranteeing asymptotic stability. In the third step, a new lemma is established for controller design using only output variables. In the fourth step, a decentralized output feedback controller is designed to force the system states to stay on the sliding surface for all time.

3.1. Single-Phase Sliding Surface Design. Let us first design a new sliding surface without reaching phase that uses only output variables and the desired motion is determined from the initial time instant. Under Assumptions 4 and 5, it follows from (11), (12), and (13) of paper [18] that there exists a 
coordinate transformation $z_{i}=T_{i} x_{i}$ such that the system (1) has the following regular form:

$$
\begin{aligned}
\dot{z}_{i}= & \left(\left[\begin{array}{cc}
A_{i 1} & A_{i 2} \\
A_{i 3} & A_{i 4}
\end{array}\right]+\left[\begin{array}{l}
D_{i 1} \\
D_{i 2}
\end{array}\right] F_{i}\left[\begin{array}{ll}
E_{i 1} & E_{i 2}
\end{array}\right]\right) z_{i} \\
& +\left[\begin{array}{c}
0 \\
B_{i 2}
\end{array}\right]\left(u_{i}+\xi_{i}\left(x_{i}, t\right)\right) \\
& +\sum_{\substack{j=1 \\
j \neq i}}^{L}\left(\left[\begin{array}{ll}
H_{i j 1} & H_{i j 2} \\
H_{i j 3} & H_{i j 4}
\end{array}\right]+\left[\begin{array}{l}
M_{i j 1} \\
M_{i j 2}
\end{array}\right] F_{i j}\left[\begin{array}{ll}
N_{i j 1} & N_{i j 2}
\end{array}\right]\right) z_{j}, \\
y_{i}= & {\left[\begin{array}{ll}
0 & C_{i 2}
\end{array}\right] z_{i}, }
\end{aligned}
$$

where

$$
\begin{gathered}
T_{i} A_{i} T_{i}^{-1}=\left[\begin{array}{ll}
A_{i 1} & A_{i 2} \\
A_{i 3} & A_{i 4}
\end{array}\right], \\
T_{i} D_{i} F_{i} E_{i} T_{i}^{-1}=\left[\begin{array}{l}
D_{i 1} \\
D_{i 2}
\end{array}\right] F_{i}\left[\begin{array}{ll}
E_{i 1} & E_{i 2}
\end{array}\right], \\
T_{i} H_{i j} T_{j}^{-1}=\left[\begin{array}{ll}
H_{i j 1} & H_{i j 2} \\
H_{i j 3} & H_{i j 4}
\end{array}\right], \quad T_{i} B_{i}=\left[\begin{array}{c}
0 \\
B_{i 2}
\end{array}\right], \\
T_{i} M_{i j} F_{i j} N_{i j} T_{j}^{-1}=\left[\begin{array}{l}
M_{i j 1} \\
M_{i j 2}
\end{array}\right] F_{i j}\left[\begin{array}{ll}
N_{i j 1} & N_{i j 2}
\end{array}\right], \\
C_{i} T_{i}^{-1}=\left[\begin{array}{ll}
0 & C_{i 2}
\end{array}\right] .
\end{gathered}
$$

The matrices $B_{i 2} \in R^{m_{i} \times m_{i}}$ and $C_{i 2} \in R^{p_{i} \times p_{i}}$ are nonsingular and $A_{i 1}=\widetilde{A}_{i 1}-\widetilde{A}_{i 2} \widetilde{K}_{i} \Xi_{i} \in R^{\left(n_{i}-m_{i}\right) \times\left(n_{i}-m_{i}\right)}$ is stable. Then, by using the sliding function (2), the sliding surface can be defined as follows:

$$
\sigma_{i}\left(x_{i}(t), t\right)=\bar{\sigma}_{i}\left(y_{i}(t), t\right)-\bar{\sigma}_{i}\left(y_{i}(t), 0\right) \exp \left(-\beta_{i} t\right)=0,
$$

where the solution of $\bar{\sigma}_{i}\left(y_{i}, t\right)$ is given by

$$
\begin{aligned}
\bar{\sigma}_{i}\left(y_{i}, t\right) & =K_{i} C_{i 2}^{-1} y_{i} \\
& =K_{i}\left[\begin{array}{cc}
N_{i} & 0_{\left(p_{i}-m_{i}\right) \times m_{i}} \\
0_{m_{i} \times\left(n_{i}-m_{i}\right)} & I_{m_{i} \times m_{i}}
\end{array}\right]\left[\begin{array}{l}
z_{i 1} \\
z_{i 2}
\end{array}\right]=K_{i 2} z_{i 2}
\end{aligned}
$$

in which $z_{i}=\left[\begin{array}{c}z_{i 1} \\ z_{i 2}\end{array}\right], z_{i 1} \in R^{n_{i}-m_{i}}, z_{i 2} \in R^{m_{i}}, N_{i}=$

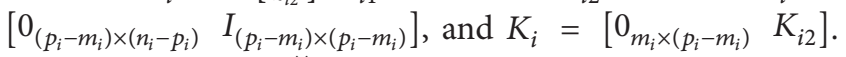
The matrix $K_{i 2} \in R^{m_{i} \times m_{i}}$ is given as

$$
K_{i 2}=\Pi_{i} P_{i} \Pi_{i}^{T},
$$

where the matrix $P_{i} \in R^{\left(n_{i}-m_{i}\right) \times\left(n_{i}-m_{i}\right)}$ is defined later and the matrix $\Pi_{i} \in R^{m_{i} \times\left(n_{i}-m_{i}\right)}$ is selected such that $K_{i 2}$ is nonsingular. Using (8), sliding surface (7) can be rewritten as

$$
\begin{aligned}
\sigma_{i}\left(x_{i}(t), t\right) & =\bar{\sigma}_{i}\left(y_{i}, t\right)-\bar{\sigma}_{i}\left(y_{i}, 0\right) \exp \left(-\beta_{i} t\right) \\
& =K_{i 2} z_{i 2}(t)-K_{i 2} z_{i 2}(0) \exp \left(-\beta_{i} t\right)=0 .
\end{aligned}
$$

Since $K_{i 2} \in R^{m_{i} \times m_{i}}$ is nonsingular, sliding surface (7) can be described by

$$
\begin{gathered}
\left\{\operatorname{col}\left(z_{1}, z_{2}, \ldots, z_{L}\right) \mid z_{i 2}=z_{i 2}(0) \exp \left(-\beta_{i} t\right),\right. \\
i=1,2, \ldots, L\} .
\end{gathered}
$$

From (11), it is clear that, in sliding mode,

$$
\dot{z}_{i 2}=-\beta_{i} z_{i 2} .
$$

Then, from the structure of system (4)-(5) and (12), the sliding mode dynamics of system (1) associated with sliding surface (7) are described by

$$
\dot{z}_{i}=\left[\begin{array}{cc}
\bar{A}_{i 1} & \bar{A}_{i 2} \\
0 & -\beta_{i} I_{m_{i} \times m_{i}}
\end{array}\right] z_{i}+\sum_{\substack{j=1 \\
j \neq i}}^{L}\left[\begin{array}{cc}
\bar{H}_{i j 1} & \bar{H}_{i j 2} \\
0 & 0
\end{array}\right] z_{j},
$$

where $\bar{A}_{i 1}=A_{i 1}+D_{i 1} F_{i} E_{i 1}, \bar{A}_{i 2}=A_{i 2}+D_{i 1} F_{i} E_{i 2}, \bar{H}_{i j 1}=$ $H_{i j 1}+M_{i j 1} F_{i j} N_{i j 1}$, and $\bar{H}_{i j 2}=H_{i j 2}+M_{i j 1} F_{i j} N_{i j 2}$.

Remark 9. It is obvious that $\sigma_{i}\left(x_{i}(0), 0\right)=0$, which means that the reaching time is equal to zero and the sliding mode exists from the initial time instant. In other words, the desired motion is determined from the beginning of the time.

Remark 10. This approach concentrates on the robustness of the motion in the entire state space. The order of the motion equation in sliding mode is equal to the order of the original system. Therefore, the robustness of the system can be assured throughout an entire response of the system starting from the initial time instance.

3.2. Single-Phase Sliding Mode Stability Analysis. Following design of the sliding surface, two tasks remain. First, for stability analysis, appropriate LMI stability conditions by the Lyapunov method must be derived to ensure the stability of sliding motion (13). Second, we design a decentralized output feedback sliding mode controller to keep the system states to stay on the sliding surface for all time.

This section focuses on the former task. We begin by considering the following LMI:

$$
\left[\begin{array}{ccc}
\Psi_{i} & P_{i} D_{i 1} & E_{i 1}^{T} \\
D_{i 1}^{T} P_{i} & -v_{i}^{-1} I & 0 \\
E_{i 1} & 0 & -\varphi_{i}^{-1} I
\end{array}\right]<0,
$$

where

$$
\begin{aligned}
\Psi_{i}= & A_{i 1}^{T} P_{i}+P_{i} A_{i 1}+\varepsilon_{i} P_{i} P_{i} \\
& +\sum_{\substack{j=1 \\
j \neq i}}^{L}\left(\widetilde{\varphi}_{j} H_{j i 1}^{T} H_{j i 1}+\widehat{\varphi}_{j} N_{j i 1}^{T} N_{j i 1}+\rho_{i} P_{i} M_{i j 1} M_{i j 1}^{T} P_{i}\right),
\end{aligned}
$$

$P_{i} \in R^{\left(n_{i}-m_{i}\right) \times\left(n_{i}-m_{i}\right)}$ is any positive matrix, and $\varphi_{i}, \varepsilon_{i}, v_{i}$, $\widehat{\varphi}_{j}, \widetilde{\varphi}_{j}, \rho_{i}$ are positive constants.

We also recall the following lemmas, which will be used in proving the stability of sliding motion (13). 
Lemma 11 (see [26]). Let $X, Y$, and $F$ be real matrices of suitable dimension with $F^{T} F \leq I$ and then, for any scalar $\varphi>0$, the following matrix inequality holds:

$$
X F Y+Y^{T} F^{T} X^{T} \leq \varphi^{-1} X X^{T}+\varphi Y^{T} Y .
$$

Lemma 12 (see [27]). Let $X$ and $Y$ be real matrices of suitable dimension and then, for any scalar $\mu>0$, the following matrix inequality holds:

$$
X^{T} Y+Y^{T} X \leq \mu X^{T} X+\mu^{-1} Y^{T} Y .
$$

Lemma 13 (see [28]). The linear matrix inequality:

$$
\left[\begin{array}{cc}
\Theta(x) & \Gamma(x) \\
\Gamma(x)^{T} & R(x)
\end{array}\right]>0
$$

where $\Theta(x)=\Theta(x)^{T}, R(x)=R(x)^{T}$, and $\Gamma(x)$ depend affinely on $x$, is equivalent to $R(x)>0, \Theta(x)-\Gamma(x) R(x)^{-1} \Gamma(x)^{T}>0$.

Then, we can establish the following theorem.

Theorem 14. Suppose that LMI (14) has a feasible solution $P_{i}>0$ and positive constants $\varphi_{i}, \varepsilon_{i}, v_{i}, \widehat{\varphi}_{j}, \widetilde{\varphi}_{j}, \rho_{i}$. And the sliding surface is given by (7). Then, the sliding motion (13) is asymptotically stable.

Proof of Theorem 14. Let us consider the following positive definition function:

$$
V=\sum_{i=1}^{L} z_{i}^{T}\left[\begin{array}{cc}
P_{i} & 0 \\
0 & \gamma_{i} Q_{i}
\end{array}\right] z_{i}
$$

where the positive constant $\gamma_{i}$ will be selected later, the positive matrix $P_{i} \in R^{\left(n_{i}-m_{i}\right) \times\left(n_{i}-m_{i}\right)}$ is defined in LMI (14), and $Q_{i} \in R^{m_{i} \times m_{i}}$ is any positive matrix. Then, taking the time derivative of $V$ along the state trajectory of system (13), we can obtain that

$$
\dot{V}=\sum_{i=1}^{L} \dot{z}_{i}^{T}\left[\begin{array}{cc}
P_{i} & 0 \\
0 & \gamma_{i} Q_{i}
\end{array}\right] z_{i}+\sum_{i=1}^{L} z_{i}^{T}\left[\begin{array}{cc}
P_{i} & 0 \\
0 & \gamma_{i} Q_{i}
\end{array}\right] \dot{z}_{i} .
$$

Hence, substituting (13) into (20), we derive

$$
\begin{aligned}
\dot{V}= & \sum_{i=1}^{L} z_{i}^{T}\left[\begin{array}{cc}
\bar{A}_{i 1}^{T} P_{i}+P_{i} \bar{A}_{i 1} & P_{i} \bar{A}_{i 2} \\
\bar{A}_{i 2}^{T} P_{i} & -2 \beta_{i} \gamma_{i} Q_{i}
\end{array}\right] z_{i} \\
& +\sum_{i=1}^{L} \sum_{\substack{j=1 \\
j \neq i}}^{L} z_{j}^{T}\left[\begin{array}{cc}
\bar{H}_{i j 1}^{T} P_{i} & 0 \\
\bar{H}_{i j 2}^{T} P_{i} & 0
\end{array}\right] z_{i} \\
& +\sum_{\substack{i=1 \\
j}}^{L} \sum_{\substack{j=1 \\
j \neq i}}^{L} z_{i}^{T}\left[\begin{array}{cc}
P_{i} \bar{H}_{i j 1} & P_{i} \bar{H}_{i j 2} \\
0 & 0
\end{array}\right] z_{j} .
\end{aligned}
$$

Since $z_{i}=\left[\begin{array}{ll}z_{i 1} & z_{i 2}\end{array}\right]^{T}, \bar{A}_{i 1}=A_{i 1}+D_{i 1} F_{i} E_{i 1}, \bar{A}_{i 2}=A_{i 2}+$ $D_{i 1} F_{i} E_{i 2}, \bar{H}_{i j 1}=H_{i j 1}+M_{i j 1} F_{i j} N_{i j 1}$, and $\bar{H}_{i j 2}=H_{i j 2}+$ $M_{i j 1} F_{i j} N_{i j 2}$, the above equation can be rewritten as

$$
\begin{gathered}
\dot{V}=\sum_{i=1}^{L} z_{i 1}^{T}\left(A_{i 1}^{T} P_{i}+E_{i 1}^{T} F_{i}^{T} D_{i 1}^{T} P_{i}\right. \\
\left.+P_{i} A_{i 1}+P_{i} D_{i 1} F_{i} E_{i 1}\right) z_{i 1} \\
+\sum_{i=1}^{L}\left[z_{i 2}^{T} A_{i 2}^{T} P_{i} z_{i 1}+z_{i 1}^{T} P_{i} A_{i 2} z_{i 2}+z_{i 2}^{T} E_{i 2}^{T} F_{i}^{T}\right. \\
\left.\cdot D_{i 1}^{T} P_{i} z_{i 1}+z_{i 1}^{T} P_{i} D_{i 1} F_{i} E_{i 2} z_{i 2}-2 \beta_{i} \gamma_{i} z_{i 2}^{T} Q_{i} z_{i 2}\right] \\
+\sum_{i=1}^{L} \sum_{\substack{j=1 \\
j \neq i}}^{L}\left(z_{j 1}^{T} H_{i j 1}^{T} P_{i} z_{i 1}+z_{j 1}^{T} N_{i j 1}^{T} F_{i j}^{T} M_{i j 1}^{T} P_{i} z_{i 1}\right. \\
\left.+z_{i 1}^{T} P_{i} H_{i j 1} z_{j 1}+z_{i 1}^{T} P_{i} M_{i j 1} F_{i j} N_{i j 1} z_{j 1}\right) \\
+\sum_{i=1}^{L} \sum_{\substack{j=1 \\
j \neq i}}^{L}\left(z_{j 2}^{T} H_{i j 2}^{T} P_{i} z_{i 1}+z_{j 2}^{T} N_{i j 2}^{T} F_{i j}^{T} M_{i j 1}^{T} P_{i} z_{i 1}\right. \\
\left.+z_{i 1}^{T} P_{i} H_{i j 2} z_{j 2}+z_{i 1}^{T} P_{i} M_{i j 1} F_{i j} N_{i j 2} z_{j 2}\right) .
\end{gathered}
$$

Using Lemma 11 and (22), we achieve

$$
\begin{aligned}
& \dot{V} \leq \sum_{i=1}^{L} z_{i 1}^{T}\left(A_{i 1}^{T} P_{i}+P_{i} A_{i 1}\right. \\
&\left.+\varphi_{i}^{-1} P_{i} D_{i 1} D_{i 1}^{T} P_{i}+\varphi_{i} E_{i 1}^{T} E_{i 1}\right) z_{i 1} \\
&+ \sum_{i=1}^{L}\left[z_{i 2}^{T} A_{i 2}^{T} P_{i} z_{i 1}+z_{i 1}^{T} P_{i} A_{i 2} z_{i 2}+\bar{\varphi}_{i} z_{i 2}^{T} E_{i 2}^{T} E_{i 2} z_{i 2}\right. \\
&\left.+\bar{\varphi}_{i}^{-1} z_{i 1}^{T} P_{i} D_{i 1} D_{i 1}^{T} P_{i} z_{i 1}-2 \beta_{i} \gamma_{i} z_{i 2}^{T} Q_{i} z_{i 2}\right] \\
&+ \sum_{i=1}^{L} \sum_{\substack{j=1 \\
j \neq i}}^{L}\left(z_{j 1}^{T} H_{i j 1}^{T} P_{i} z_{i 1}+z_{i 1}^{T} P_{i} H_{i j 1} z_{j 1}+\widehat{\varphi}_{i} z_{j 1}^{T} N_{i j 1}^{T}\right. \\
&+\sum_{i=1}^{L} \sum_{\substack{j=1 \\
j \neq i}}^{L}\left(z_{j 2}^{T} H_{i j 2}^{T} P_{i} z_{i 1}+z_{i 1}^{T} P_{i} H_{i j 2} z_{j 2}+\widehat{\delta}_{i} z_{j 2}^{T} N_{i j 2}^{T}\right. \\
&\left.\cdot N_{i j 2} z_{j 2}+\widehat{\delta}_{i}^{-1} z_{i 1}^{T} P_{i} M_{i j 1} M_{i j 1}^{T} P_{i} z_{i 1}\right)
\end{aligned}
$$

where $\varphi_{i}>0, \bar{\varphi}_{i}>0, \widehat{\varphi}_{i}>0$ and $\widehat{\delta}_{i}>0$ are scalars. Applying Lemma 12 to (23), we can obtain 


$$
\begin{aligned}
& \dot{V} \leq \sum_{i=1}^{L} z_{i 1}^{T}\left(A_{i 1}^{T} P_{i}+P_{i} A_{i 1}+\varphi_{i}^{-1} P_{i} D_{i 1} D_{i 1}^{T} P_{i}+\varphi_{i} E_{i 1}^{T} E_{i 1}\right) z_{i 1} \\
& +\sum_{i=1}^{L}\left[\delta_{i} z_{i 2}^{T} A_{i 2}^{T} A_{i 2} z_{i 2}+\delta_{i}^{-1} z_{i 1}^{T} P_{i} P_{i} z_{i 1}+\bar{\varphi}_{i} z_{i 2}^{T} E_{i 2}^{T} E_{i 2} z_{i 2}\right. \\
& \left.+\bar{\varphi}_{i}^{-1} z_{i 1}^{T} P_{i} D_{i 1} D_{i 1}^{T} P_{i} z_{i 1}-2 \beta_{i} \gamma_{i} z_{i 2}^{T} Q_{i} z_{i 2}\right] \\
& +\sum_{i=1}^{L} \sum_{\substack{j=1 \\
j \neq i}}^{L}\left(\widetilde{\varphi}_{i} z_{j 1}^{T} H_{i j 1}^{T} H_{i j 1} z_{j 1}+\widetilde{\varphi}_{i}^{-1} z_{i 1}^{T} P_{i} P_{i} z_{i 1}+\widehat{\varphi}_{i} z_{j 1}^{T} N_{i j 1}^{T}\right. \\
& \left.\cdot N_{i j 1} z_{j 1}+\widehat{\varphi}_{i}^{-1} z_{i 1}^{T} P_{i} M_{i j 1} M_{i j 1}^{T} P_{i} z_{i 1}\right) \\
& +\sum_{i=1}^{L} \sum_{\substack{j=1 \\
j \neq i}}^{L}\left(\widetilde{\delta}_{i} z_{j 2}^{T} H_{i j 2}^{T} H_{i j 2} z_{j 2}+\widetilde{\delta}_{i}^{-1} z_{i 1}^{T} P_{i} P_{i} z_{i 1}+\widehat{\delta}_{i} z_{j 2}^{T} N_{i j 2}^{T}\right. \\
& \left.\cdot N_{i j 2} z_{j 2}+\widehat{\delta}_{i}^{-1} z_{i 1}^{T} P_{i} M_{i j 1} M_{i j 1}^{T} P_{i} z_{i 1}\right),
\end{aligned}
$$

where $\widetilde{\varphi}_{i}>0, \delta_{i}>0$ and $\widetilde{\delta}_{i}>0$ are scalars. From (24) and property

$$
\begin{aligned}
& \sum_{i=1}^{L} \sum_{\substack{j=1 \\
j \neq i}}^{L}\left(\widetilde{\varphi}_{i} z_{j 1}^{T} H_{i j 1}^{T} H_{i j 1} z_{j 1}+\widehat{\varphi}_{i} z_{j 1}^{T} N_{i j 1}^{T} N_{i j 1} z_{j 1}\right) \\
& \quad=\sum_{\substack { i=1 \\
\begin{subarray}{c}{j=1 \\
j \neq i{ i = 1 \\
\begin{subarray} { c } { j = 1 \\
j \neq i } }\end{subarray}}^{L}\left(\widetilde{\varphi}_{j} z_{i 1}^{T} H_{j i 1}^{T} H_{j i 1} z_{i 1}+\widehat{\varphi}_{j} z_{i 1}^{T} N_{j i 1}^{T} N_{j i 1} z_{i 1}\right), \\
& \sum_{i=1}^{L} \sum_{\substack{j=1 \\
j \neq i}}^{L}\left(\widetilde{\delta}_{i} z_{j 2}^{T} H_{i j 2}^{T} H_{i j 2} z_{j 2}+\widehat{\delta}_{i} z_{j 2}^{T} N_{i j 2}^{T} N_{i j 2} z_{j 2}\right) \\
& \quad=\sum_{i=1}^{L} \sum_{\substack{j=1 \\
j \neq i}}^{L}\left(\widetilde{\delta}_{j} z_{i 2}^{T} H_{j i 2}^{T} H_{j i 2} z_{i 2}+\widehat{\delta}_{j} z_{i 2}^{T} N_{j i 2}^{T} N_{j i 2} z_{i 2}\right),
\end{aligned}
$$

it generates

$$
\begin{aligned}
& \dot{V} \leq \sum_{i=1}^{L} z_{i 1}^{T}\left[A_{i 1}^{T} P_{i}+P_{i} A_{i 1}+\varphi_{i} E_{i 1}^{T} E_{i 1}\right. \\
& +\varepsilon_{i} P_{i} P_{i}+v_{i} P_{i} D_{i 1} D_{i 1}^{T} P_{i} \\
& +\sum_{\substack{j=1 \\
j \neq i}}^{L}\left(\widetilde{\varphi}_{j} H_{j i 1}^{T} H_{j i 1}+\widehat{\varphi}_{j} N_{j i 1}^{T} N_{j i 1}\right. \\
& \left.\left.+\rho_{i} P_{i} M_{i j 1} M_{i j 1}^{T} P_{i}\right)\right] z_{i 1}
\end{aligned}
$$

$$
\begin{aligned}
& +\sum_{i=1}^{L} z_{i 2}^{T}\left[-2 \beta_{i} \gamma_{i} Q_{i}+\delta_{i} A_{i 2}^{T} A_{i 2}+\bar{\varphi}_{i} E_{i 2}^{T} E_{i 2}\right. \\
& \left.+\sum_{\substack{j=1 \\
j \neq i}}^{L}\left(\widetilde{\delta}_{j} H_{j i 2}^{T} H_{j i 2}+\widehat{\delta}_{j} N_{j i 2}^{T} N_{j i 2}\right)\right] z_{i 2},
\end{aligned}
$$

where $v_{i}=\varphi_{i}^{-1}+\bar{\varphi}_{i}^{-1}, \rho_{i}=\widehat{\varphi}_{i}^{-1}+\widehat{\delta}_{i}^{-1}$, and $\varepsilon_{i}=(L-1) \widetilde{\varphi}_{i}^{-1}+$ $\delta_{i}^{-1}+(L-1) \widetilde{\delta}_{i}^{-1}$ and $L$ is the number of subsystems. Let us define $\Omega_{i}=2 \beta_{i} Q_{i}>0$, so that all the eigenvalues of $\Omega_{i}$ are greater than some positive $\ni_{i}$. Then, it is easy to select

$$
\begin{aligned}
& \gamma_{i}>\frac{1}{\ni_{i}} \max _{\substack{i=1: L \\
j=1 \\
i \neq j}}\left[\delta_{i}\left\|A_{i 2}^{T} A_{i 2}\right\|+\bar{\varphi}_{i}\left\|E_{i 2}^{T} E_{i 2}\right\|\right. \\
& \left.+\sum_{\substack{j=1 \\
j \neq i}}^{L}\left(\widetilde{\delta}_{j}\left\|H_{j i 2}^{T} H_{j i 2}\right\|+\widehat{\delta}_{j}\left\|N_{j i 2}^{T} N_{j i 2}\right\|\right)\right] .
\end{aligned}
$$

According to (27) and the result of paper [29], we have

$$
\begin{aligned}
& -2 \beta_{i} \gamma_{i} Q_{i}+\delta_{i} A_{i 2}^{T} A_{i 2}+\bar{\varphi}_{i} E_{i 2}^{T} E_{i 2} \\
& +\sum_{\substack{j=1 \\
j \neq i}}^{L}\left(\widetilde{\delta}_{j} H_{j i 2}^{T} H_{j i 2}+\widehat{\delta}_{j} N_{i j 2}^{T} N_{j i 2}\right)<0 .
\end{aligned}
$$

By applying Lemma 13, LMI (14) is equivalent to the following inequality:

$$
\begin{aligned}
& A_{i 1}^{T} P_{i}+P_{i} A_{i 1}+\varphi_{i} E_{i 1}^{T} E_{i 1}+\varepsilon_{i} P_{i} P_{i}+v_{i} P_{i} D_{i 1} D_{i 1}^{T} P_{i} \\
& \quad+\sum_{\substack{j=1 \\
j \neq i}}^{L}\left(\widetilde{\varphi}_{j} H_{j i 1}^{T} H_{j i 1}+\widehat{\varphi}_{j} N_{j i 1}^{T} N_{j i 1}+\rho_{i} P_{i} M_{i j 1} M_{i j 1}^{T} P_{i}\right)<0 .
\end{aligned}
$$

From (26), (28), and (29), we have

$$
\dot{V}<0 .
$$

Inequality (30) implies that if LMI (14) holds, then sliding motion (13) is asymptotically stable.

Remark 15. Theorem 14 provides an existence condition of the sliding surface in terms of strict LMI, which can be easily worked out using the LMI Toolbox in MATLAB.

Remark 16. It is seen that, compared to the the recent LMI methods [17], the present LMI method shows less conservative results and easily finds a feasible solution of the LMI. 
In order to design a new output feedback sliding mode control scheme for complex interconnected system (1), we establish the following lemma.

Lemma 17. Consider a class of interconnected systems that is decomposed into L subsystems

$$
\begin{aligned}
\dot{v}_{i}= & {\left[S_{i}+G_{i} \Delta_{i}\left(v_{i}, t\right) X_{i}\right] v_{i}+B_{i}\left(u_{i}+\xi_{i}\left(v_{i}, t\right)\right) } \\
& +\sum_{\substack{j=1 \\
j \neq i}}^{L}\left(A_{i j}+D_{i j} \Delta_{i j}\left(v_{j}, t\right) E_{i j}\right) v_{j},
\end{aligned}
$$

where $v_{i}=\left[\begin{array}{l}v_{i 1} \\ v_{i 2}\end{array}\right]$ are the state variables of the ith subsystem with $v_{i 1} \in R^{n_{i}-m_{i}}$ and $v_{i 2} \in R^{m_{i}}$. The matrices $S_{i}=\left[\begin{array}{ll}S_{i 1} & S_{i 2} \\ S_{i 3} & S_{i 4}\end{array}\right]$, $G_{i}=\left[\begin{array}{l}G_{i 1} \\ G_{i 2}\end{array}\right], X_{i}=\left[\begin{array}{ll}X_{i 1} & X_{i 2}\end{array}\right], B_{i}=\left[\begin{array}{c}0 \\ B_{i 2}\end{array}\right], A_{i j}=\left[\begin{array}{ll}A_{i j 1} & A_{i j 2} \\ A_{i j 3} & A_{i j 4}\end{array}\right]$, $D_{i j}=\left[\begin{array}{l}D_{i j 1} \\ D_{i j 2}\end{array}\right]$, and $E_{i j}=\left[\begin{array}{ll}E_{i j 1} & E_{i j 2}\end{array}\right]$ are known matrices of appropriate dimensions. The matrices $\Delta_{i}\left(v_{i}, t\right)$ and $\Delta_{i j}\left(v_{j}, t\right)$ are unknown but bounded as $\left\|\Delta_{i}\left(v_{i}, t\right)\right\| \leq 1$ and $\left\|\Delta_{i j}\left(v_{j}, t\right)\right\| \leq$ 1. If the matrix $S_{i 1}$ is stable, then $\sum_{i=1}^{L}\left\|v_{i 1}(t)\right\|$ is bounded by $\sum_{i=1}^{L} \phi_{i}(t)$ for all time, where $\phi_{i}(t)$ is the solution of

$$
\begin{array}{r}
\dot{\phi}_{i}(t)=\widehat{k}_{i} \phi_{i}(t)+k_{i}\left[\left\|S_{i 2}\right\|+\left\|G_{i 1}\right\|\left\|X_{i 2}\right\|\right. \\
\left.+\sum_{\substack{j=1 \\
j \neq i}}^{L}\left(\left\|A_{j i 2}\right\|+\left\|D_{j i 2}\right\|\left\|E_{j i 2}\right\|\right)\right]\left\|v_{i 2}\right\|, \\
i=1,2, \ldots, L,
\end{array}
$$

in which $\hat{k}_{i}=k_{i}\left[\left\|G_{i 1}\right\|\left\|X_{i 1}\right\|+\sum_{j=1, j \neq i}^{L}\left(\left\|A_{j i 1}\right\|+\right.\right.$ $\left.\left.\left\|D_{j i 1}\right\|\left\|E_{j i 1}\right\|\right)\right]+\lambda_{i}<0, k_{i}>0 . \lambda_{i}$ is the maximum eigenvalue of the matrix $S_{i 1}$.

Proof of Lemma 17. We are now in the position to prove Lemma 17. From (31), it is obvious that

$$
\begin{gathered}
\dot{v}_{i 1}(t)=\left(S_{i 1}+G_{i 1} \Delta_{i} X_{i 1}\right) v_{i 1}+\left(S_{i 2}+G_{i 1} \Delta_{i} X_{i 2}\right) v_{i 2} \\
+\sum_{\substack{j=1 \\
j \neq i}}^{L}\left[\left(A_{i j 1}+D_{i j 1} \Delta_{i j} E_{i j 1}\right) v_{j 1}\right. \\
\left.+\left(A_{i j 2}+D_{i j 1} \Delta_{i j} E_{i j 2}\right) v_{j 2}\right] .
\end{gathered}
$$

According to (33), we can obtain

$$
\begin{aligned}
& \left\|v_{i 1}(t)\right\| \leq\left\|\exp \left(S_{i 1} t\right)\right\|\left\|v_{i 1}(0)\right\| \\
& +\int_{0}^{t}\left\|\exp \left(S_{i 1}(t-\tau)\right)\right\| \\
& \cdot\left(\left\|S_{i 2}\right\|+\left\|G_{i 1}\right\|\left\|X_{i 2}\right\|\right)\left\|v_{i 2}\right\| d \tau \\
& +\int_{0}^{t}\left\|\exp \left(S_{i 1}(t-\tau)\right)\right\| \\
& \cdot\left[\left\|G_{i 1}\right\|\left\|X_{i 1}\right\|\left\|v_{i 1}\right\|\right. \\
& +\sum_{\substack{j=1 \\
j \neq i}}^{L}\left(\left\|A_{i j 1}\right\|+\left\|D_{i j 1}\right\|\left\|E_{i j 1}\right\|\right)\left\|v_{j 1}\right\| \\
& \left.+\sum_{\substack{j=1 \\
j \neq i}}^{L}\left(\left\|A_{i j 2}\right\|+\left\|D_{i j 1}\right\|\left\|E_{i j 2}\right\|\right)\left\|v_{j 2}\right\|\right] d \tau .
\end{aligned}
$$

The stable matrix $S_{i 1}$ implies that $\left\|\exp \left(S_{i 1} t\right)\right\| \leq k_{i} \exp \left(\lambda_{i} t\right)$, for some $k_{i}>0$, and the inequality (34) can be rewritten as

$$
\begin{gathered}
\left\|v_{i 1}\right\| \leq k_{i} \exp \left(\lambda_{i} t\right)\left\|v_{i 1}(0)\right\| \\
+\int_{0}^{t} k_{i} \exp \left(\lambda_{i}(t-\tau)\right) \\
\cdot\left[\left\|G_{i 1}\right\|\left\|X_{i 1}\right\|\left\|v_{i 1}\right\|\right. \\
\left.+\left(\left\|S_{i 2}\right\|+\left\|G_{i 1}\right\|\left\|X_{i 2}\right\|\right)\left\|v_{i 2}\right\|\right] d \tau \\
+\int_{0}^{t} k_{i} \exp \left(\lambda_{i}(t-\tau)\right) \\
\cdot\left[\sum_{\substack{j=1 \\
j \neq i}}^{L}\left(\left\|A_{i j 1}\right\|+\left\|D_{i j 1}\right\|\left\|E_{i j 1}\right\|\right)\left\|v_{j 1}\right\|\right. \\
\left.+\sum_{\substack{j=1 \\
j \neq i}}^{L}\left(\left\|A_{i j 2}\right\|+\left\|D_{i j 1}\right\|\left\|E_{i j 2}\right\|\right)\left\|v_{j 2}\right\|\right] d \tau .
\end{gathered}
$$

For the above inequality, we multiply both sides by the term $\exp \left(-\lambda_{i} t\right)$ 


$$
\begin{aligned}
& \left\|v_{i 1}(t)\right\| \exp \left(-\lambda_{i} t\right) \\
& \leq k_{i}\left\|v_{i 1}(0)\right\| \\
& +\int_{0}^{t} k_{i} \exp \left(-\lambda_{i} \tau\right) \\
& \cdot\left[\left\|G_{i 1}\right\|\left\|X_{i 1}\right\|\left\|v_{i 1}\right\|+\left(\left\|S_{i 2}\right\|+\left\|G_{i 1}\right\|\left\|X_{i 2}\right\|\right)\left\|v_{i 2}\right\|\right] d \tau \\
& +\int_{0}^{t} k_{i} \exp \left(-\lambda_{i} \tau\right) \\
& \cdot\left[\sum_{\substack{j=1 \\
j \neq i}}^{L}\left(\left\|A_{i j 1}\right\|+\left\|D_{i j 1}\right\|\left\|E_{i j 1}\right\|\right)\left\|v_{j 1}\right\|\right. \\
& \left.+\sum_{\substack{j=1 \\
j \neq i}}^{L}\left(\left\|A_{i j 2}\right\|+\left\|D_{i j 1}\right\|\left\|E_{i j 2}\right\|\right)\left\|v_{j 2}\right\|\right] d \tau .
\end{aligned}
$$

Let $s_{i}(t)$ represent the right side of the inequality (36)

$$
\begin{gathered}
s_{i}(t)=k_{i}\left\|v_{i 1}(0)\right\| \\
+\int_{0}^{t} k_{i} \exp \left(-\lambda_{i} \tau\right) \\
\cdot\left[\left\|G_{i 1}\right\|\left\|X_{i 1}\right\|\left\|v_{i 1}\right\|\right. \\
\left.+\left(\left\|S_{i 2}\right\|+\left\|G_{i 1}\right\|\left\|X_{i 2}\right\|\right)\left\|v_{i 2}\right\|\right] d \tau \\
+\int_{0}^{t} k_{i} \exp \left(-\lambda_{i} \tau\right) \\
\cdot\left[\sum_{\substack{j=1 \\
j \neq i}}^{L}\left(\left\|A_{i j 1}\right\|+\left\|D_{i j 1}\right\|\left\|E_{i j 1}\right\|\right)\left\|v_{j 1}\right\|\right. \\
\left.+\sum_{\substack{j=1 \\
j \neq i}}^{L}\left(\left\|A_{i j 2}\right\|+\left\|D_{i j 1}\right\|\left\|E_{i j 2}\right\|\right)\left\|v_{j 2}\right\|\right] d \tau .
\end{gathered}
$$

Hence, by taking the time derivative of $s_{i}(t)$, we can obtain

$$
\begin{aligned}
& \frac{1}{k_{i}} \exp \left(\lambda_{i} t\right) \frac{d}{d t} s_{i}(t) \\
& =\left(\left\|S_{i 2}\right\|+\left\|G_{i 1}\right\|\left\|X_{i 2}\right\|\right)\left\|v_{i 2}\right\|+\left\|G_{i 1}\right\|\left\|X_{i 1}\right\|\left\|v_{i 1}\right\| \\
& \quad+\sum_{\substack{j=1 \\
j \neq i}}^{L}\left(\left\|A_{i j 1}\right\|+\left\|D_{i j 1}\right\|\left\|E_{i j 1}\right\|\right)\left\|v_{j 1}\right\| \\
& +\sum_{\substack{j=1 \\
j \neq i}}^{L}\left(\left\|A_{i j 2}\right\|+\left\|D_{i j 1}\right\|\left\|E_{i j 2}\right\|\right)\left\|v_{j 2}\right\| .
\end{aligned}
$$

Then, by taking the summation $i=1,2, \ldots, L$ to both sides of (38), we have

$$
\begin{aligned}
& \sum_{i=1}^{L} \frac{1}{k_{i}} \exp \left(\lambda_{i} t\right) \frac{d}{d t} s_{i}(t) \\
& =\sum_{i=1}^{L}\left(\left\|S_{i 2}\right\|+\left\|G_{i 1}\right\|\left\|X_{i 2}\right\|\right)\left\|v_{i 2}\right\| \\
& \quad+\sum_{i=1}^{L}\left\|G_{i 1}\right\|\left\|X_{i 1}\right\|\left\|v_{i 1}\right\| \\
& \quad+\sum_{i=1}^{L} \sum_{\substack{j=1 \\
j \neq i}}^{L}\left(\left\|A_{j i 2}\right\|+\left\|D_{j i 1}\right\|\left\|E_{j i 2}\right\|\right)\left\|v_{i 2}\right\| \\
& \quad+\sum_{\substack { i=1 \\
\begin{subarray}{c}{j=1 \\
j \neq i{ i = 1 \\
\begin{subarray} { c } { j = 1 \\
j \neq i } }\end{subarray}}^{L}\left(\left\|A_{j i 1}\right\|+\left\|D_{j i 1}\right\|\left\|E_{j i 1}\right\|\right)\left\|v_{i 1}\right\| .
\end{aligned}
$$

For the above equation, we multiply both sides by the term $k_{i} \exp \left(-\lambda_{i} t\right)$. Since $\left\|v_{i 1}(t)\right\| \exp \left(-\lambda_{i} t\right) \leq s_{i}(t)$, one can get that

$$
\begin{gathered}
\sum_{i=1}^{L} \frac{d}{d t} s_{i}(t) \leq \sum_{i=1}^{L} k_{i} \exp \left(-\lambda_{i} t\right) \\
\cdot\left[\left\|S_{i 2}\right\|+\left\|G_{i 1}\right\|\left\|X_{i 2}\right\|\right. \\
\left.+\sum_{\substack{j=1 \\
j \neq i}}^{L}\left(\left\|A_{j i 2}\right\|+\left\|D_{j i 1}\right\|\left\|E_{j i 2}\right\|\right)\right]\left\|v_{i 2}\right\| \\
+\sum_{i=1}^{L} \bar{k}_{i} s_{i}(t),
\end{gathered}
$$

where $\bar{k}_{i}=k_{i}\left(\left\|G_{i 1}\right\|\left\|X_{i 1}\right\|+\sum_{j=1, j \neq i}^{L}\left(\left\|A_{j i 1}\right\|+\left\|D_{j i 1}\right\|\left\|E_{j i 1}\right\|\right)\right)$. We multiply the term $\exp \left(-\bar{k}_{i} t\right)$ to both sides of the inequality (40), and then

$$
\begin{gathered}
\sum_{i=1}^{L} \frac{d}{d t}\left[s_{i}(t) \exp \left(-\bar{k}_{i} t\right)\right] \\
\leq \sum_{i=1}^{L} k_{i} \exp \left(-\lambda_{i} t\right)
\end{gathered}
$$




$$
\begin{aligned}
& {\left[\left\|S_{i 2}\right\|+\left\|G_{i 1}\right\|\left\|X_{i 2}\right\|\right.} \\
& \left.+\sum_{\substack{j=1 \\
j \neq i}}^{L}\left(\left\|A_{j i 2}\right\|+\left\|D_{j i 1}\right\|\left\|E_{j i 2}\right\|\right)\right]\left\|v_{i 2}\right\| \exp \left(-\bar{k}_{i} t\right) .
\end{aligned}
$$

Integrating inequality (41) on both sides, we obtain

$$
\begin{gathered}
\sum_{i=1}^{L} s_{i}(t) \leq \sum_{i=1}^{L} k_{i}\left\|v_{i 1}(0)\right\| \exp \left(\bar{k}_{i} t\right) \\
+\sum_{i=1}^{L}\left\{\int_{0}^{t} k_{i} \exp \left(-\lambda_{i} \tau\right)\right. \\
\cdot\left[\begin{array}{l}
\left\|S_{i 2}\right\|+\left\|G_{i 1}\right\|\left\|X_{i 2}\right\| \\
+\sum_{\substack{j=1 \\
j \neq i}}^{L}\left(\left\|A_{j i 2}\right\|+\left\|D_{j i 1}\right\|\left\|E_{j i 2}\right\|\right) \\
\left.\cdot\left\|v_{i 2}\right\| \exp \left(-\bar{k}_{i} \tau\right) d \tau\right\} \exp \left(\bar{k}_{i} t\right) .
\end{array}\right.
\end{gathered}
$$

Since $\left\|v_{i 1}(t)\right\| \exp \left(-\lambda_{i} t\right) \leq s_{i}(t)$, we can show that

$$
\begin{aligned}
& \sum_{i=1}^{L}\left\|v_{i 1}(t)\right\| \leq \sum_{i=1}^{L}\left\{\phi_{i}(0) \exp \left(\left(\bar{k}_{i}+\lambda_{i}\right) t\right)\right. \\
& +\int_{0}^{t} k_{i} \exp \left[\left(\bar{k}_{i}+\lambda_{i}\right)(t-\tau)\right] \\
& \times\left[\left\|S_{i 2}\right\|+\left\|G_{i 1}\right\|\left\|X_{i 2}\right\|\right. \\
& \left.+\sum_{\substack{j=1 \\
j \neq i}}^{L}\left(\left\|A_{j i 2}\right\|+\left\|D_{j i 1}\right\|\left\|E_{j i 2}\right\|\right)\right]
\end{aligned}
$$

$$
\begin{gathered}
\left.\cdot\left\|v_{i 2}\right\| d \tau\right\} \\
=\sum_{i=1}^{L} \phi_{i}(t) \quad \text { if } \phi_{i}(0) \geq k_{i}\left\|v_{i 1}(0)\right\|>0,
\end{gathered}
$$

where the time function $\phi_{i}(t)$ satisfies (32). Hence, we can see that $\sum_{i=1}^{L} \phi_{i}(t) \geq \sum_{i=1}^{L}\left\|v_{i 1}(t)\right\|$ for all time, if $\phi_{i}(0)$ is sufficiently large.

Remark 18. It is obvious that the time function $\phi_{i}(t)$ is only dependent on the state variable $v_{i 2}$. Therefore, the term $\sum_{i=1}^{L}\left\|v_{i 1}\right\|$ is bounded by a function of state variable $v_{i 2}$. This feature is useful in the design of a controller, which only uses output variables.

3.3. Decentralized Output Feedback Single-Phase Sliding Mode Controller Design. In the last section, we proved that the sliding motion (13) is asymptotically stable. We further established Lemma 17. Now, by applying this lemma, we design a decentralized output feedback controller to keep the system states to stay in the sliding surface for all time. This is achieved when the following two conditions are satisfied: (1) reaching time is equal to zero $\left(\sigma_{i}\left(x_{i}(0), 0\right)=0\right)$; (2) the reaching conditions are satisfied by the Lyapunov function $V\left(\sigma_{i}\left(x_{i}(t), t\right)\right)>0$ and $\dot{V}\left(\sigma_{i}\left(x_{i}(t), t\right)\right)<0$ holds for all $t \geq 0$. Sliding surface (7) allows for the first condition to be met. In order to prove the second condition is also satisfied, the single-phase sliding mode controller is selected to be

$$
u_{i}(t)=-\left(K_{i 2} B_{i 2}\right)^{-1}\left(\widehat{\kappa}_{i}+\kappa_{i}\left\|y_{i}\right\|+\bar{\kappa}_{i} \eta_{i}\right) \frac{\sigma_{i}}{\left\|\sigma_{i}\right\|},
$$

where

$$
\begin{aligned}
\bar{\kappa}_{i}= & \left\|K_{i 2}\right\|\left(\left\|A_{i 3}\right\|+\left\|D_{i 2}\right\|\left\|E_{i 1}\right\|+b_{i}\left\|B_{i 2}\right\|\left\|W_{i 1}\right\|\right) \\
& +\sum_{\substack{j=1 \\
j \neq i}}^{L}\left\|K_{j 2}\right\|\left(\left\|H_{j i 3}\right\|+\left\|M_{j i 2}\right\|\left\|N_{j i 1}\right\|\right), \\
\kappa_{i}= & {\left[\left\|K_{i 2}\right\|\left(\left\|A_{i 4}\right\|+\left\|D_{i 2}\right\|\left\|E_{i 2}\right\|+b_{i}\left\|B_{i 2}\right\|\left\|W_{i 2}\right\|\right)\right.} \\
& \left.+\sum_{\substack{j=1 \\
j \neq i}}^{L}\left\|K_{j 2}\right\|\left(\left\|H_{j i 2}\right\|+\left\|M_{j i 2}\right\|\left\|N_{j i 2}\right\|\right)\right]
\end{aligned}
$$




$$
\begin{gathered}
\cdot\left\|K_{i 2}^{-1}\right\|\left\|K_{i} C_{i 2}^{-1}\right\|, \\
\widehat{\kappa}_{i}=\beta_{i}\left\|K_{i} C_{i 2}^{-1}\right\|\left\|y_{i}(0)\right\| \exp \left(-\beta_{i} t\right) \\
+\alpha_{i}+c_{i}\left\|K_{i 2}\right\|\left\|B_{i 2}\right\|,
\end{gathered}
$$

$\left[\begin{array}{ll}W_{i 1} & W_{i 2}\end{array}\right]=T_{i}^{-1}$,

and the scalar $\alpha_{i}>0$ and $\eta_{i}(t)$ is the solution of

$$
\begin{aligned}
& \dot{\eta}_{i}(t)=\widehat{k}_{i} \eta_{i}(t) \\
&+k_{i}\left[\left\|A_{i 2}\right\|+\left\|D_{i 1}\right\|\left\|E_{i 2}\right\|\right. \\
&\left.+\sum_{\substack{j=1 \\
j \neq i}}^{L}\left(\left\|H_{j i 2}\right\|+\left\|M_{j i 1}\right\|\left\|N_{j i 2}\right\|\right)\right] \\
& \cdot\left\|K_{i 2}^{-1}\right\|\left\|K_{i} C_{i 2}^{-1}\right\|\left\|y_{i}\right\|
\end{aligned}
$$

in which $\hat{k}_{i}=k_{i}\left[\left\|D_{i 1}\right\|\left\|E_{i 1}\right\|+\sum_{j=1, j \neq i}^{L}\left(\left\|H_{j i 1}\right\|+\right.\right.$ $\left.\left.\left\|M_{j i 1}\right\|\left\|N_{j i 1}\right\|\right)\right]+\lambda_{i}<0, k_{i}>0$, and $\lambda_{i}$ is the maximum eigenvalue of the matrix $A_{i 1}$. It should be pointed out that controller (44) uses only output variables.

Now, we can establish the following theorem.

Theorem 19. Suppose that LMI (14) has a feasible solution $P_{i}>0$ and positive constants $\varphi_{i}, \varepsilon_{i}, v_{i}, \widehat{\varphi}_{j}, \widetilde{\varphi}_{j}, \rho_{i}$. Consider the closed loop of the system (1) with the above-decentralized output feedback controller (44), where the sliding surface is given by (7). Then, the system states stay on the sliding surface for all time.

Proof of Theorem 19. Now, we are going to prove Theorem 19. Let us consider the following Lyapunov function:

$$
V\left(\sigma_{i}\left(x_{i}(t), t\right)\right)=\sum_{i=1}^{L}\left\|\sigma_{i}\right\| .
$$

By differentiating (47) along the trajectories of (7), we can obtain

$$
\begin{aligned}
\dot{V} & =\sum_{i=1}^{L} \frac{\sigma_{i}^{T}}{\left\|\sigma_{i}\right\|} \dot{\sigma}_{i} \\
& =\sum_{i=1}^{L} \frac{\sigma_{i}^{T}}{\left\|\sigma_{i}\right\|}\left(K_{i 2} \dot{z}_{i 2}+\beta_{i} K_{i} C_{i 2}^{-1} y_{i}(0) \exp \left(-\beta_{i} t\right)\right) .
\end{aligned}
$$

From (4), it is clear that

$$
\begin{aligned}
\dot{z}_{i 2}= & \left(A_{i 3}+D_{i 2} F_{i} E_{i 1}\right) z_{i 1}+B_{i 2}\left(u_{i}+\xi_{i}\right) \\
& +\left(A_{i 4}+D_{i 2} F_{i} E_{i 2}\right) z_{i 2} \\
& +\sum_{\substack{j=1 \\
j \neq i}}^{L}\left[\left(H_{i j 3}+M_{i j 2} F_{i j} N_{i j 1}\right) z_{j 1}\right. \\
& \left.+\left(H_{i j 4}+M_{i j 2} F_{i j} N_{i j 2}\right) z_{j 2}\right] .
\end{aligned}
$$

According to (48) and (49), we have

$$
\begin{aligned}
& \dot{V}=\sum_{i=1}^{L} \frac{\sigma_{i}^{T}}{\left\|\sigma_{i}\right\|}\left[K_{i 2}\left(A_{i 3}+D_{i 2} F_{i} E_{i 1}\right) z_{i 1}\right. \\
& +K_{i 2}\left(A_{i 4}+D_{i 2} F_{i} E_{i 2}\right) z_{i 2} \\
& \left.+\beta_{i} K_{i} C_{i 2}^{-1} y_{i}(0) \exp \left(-\beta_{i} t\right)\right] \\
& +\sum_{i=1}^{L} \frac{\sigma_{i}^{T}}{\left\|\sigma_{i}\right\|} K_{i 2} B_{i 2}\left(u_{i}+\xi_{i}\left(x_{i}, t\right)\right) \\
& +\sum_{i=1}^{L} \sum_{\substack{j=1 \\
j \neq i}}^{L} \frac{\sigma_{i}^{T}}{\left\|\sigma_{i}\right\|} K_{i 2}\left(H_{i j 4}+M_{i j 2} F_{i j} N_{i j 2}\right) z_{j 2} \\
& +\sum_{i=1}^{L} \sum_{\substack{j=1 \\
j \neq i}}^{L} \frac{\sigma_{i}^{T}}{\left\|\sigma_{i}\right\|} K_{i 2}\left(H_{i j 3}+M_{i j 2} F_{i j} N_{i j 1}\right) z_{j 1} .
\end{aligned}
$$

Using (50) and property $\|A B\| \leq\|A\|\|B\|$, we can generate

$$
\begin{gathered}
\dot{V} \leq \sum_{i=1}^{L}\left[\left\|K_{i 2}\right\|\left(\left\|A_{i 3}\right\|+\left\|D_{i 2}\right\|\left\|E_{i 1}\right\|\right)\left\|z_{i 1}\right\|\right. \\
+\left\|K_{i 2}\right\|\left(\left\|A_{i 4}\right\|+\left\|D_{i 2}\right\|\left\|E_{i 2}\right\|\right)\left\|z_{i 2}\right\| \\
\left.+\beta_{i}\left\|K_{i} C_{i 2}^{-1}\right\|\left\|y_{i}(0)\right\| \exp \left(-\beta_{i} t\right)\right] \\
+\sum_{i=1}^{L} \sum_{\substack{j=1 \\
j \neq i}}^{L}\left\|K_{i 2}\right\|\left(\left\|H_{i j 4}\right\|+\left\|M_{i j 2}\right\|\left\|N_{i j 2}\right\|\right)\left\|z_{j 2}\right\| \\
+\sum_{i=1}^{L} \sum_{\substack{j=1 \\
j \neq i}}^{L}\left\|K_{i 2}\right\|\left(\left\|H_{i j 3}\right\|+\left\|M_{i j 2}\right\|\left\|N_{i j 1}\right\|\right)\left\|z_{j 1}\right\| \\
+\sum_{i=1}^{L} \frac{\sigma_{i}^{T}}{\left\|\sigma_{i}\right\|} K_{i 2} B_{i 2} u_{i}+\sum_{i=1}^{L}\left\|K_{i 2}\right\|\left\|B_{i 2}\right\|\left\|\xi_{i}\right\| .
\end{gathered}
$$


Since

$$
\begin{aligned}
& \sum_{\substack{i=1 \\
j \neq i}}^{L} \sum_{\substack{j=1 \\
j}}^{L}\left\|\left(\left\|H_{j i 4}\right\|+\left\|M_{j i 2}\right\|\left\|N_{j i 2}\right\|\right)\right\| z_{i 2} \| \\
& =\sum_{\substack { i=1 \\
\begin{subarray}{c}{j=1 \\
j \neq i{ i = 1 \\
\begin{subarray} { c } { j = 1 \\
j \neq i } }\end{subarray}}^{L}\left\|K_{i 2}\right\|\left(\left\|H_{i j 4}\right\|+\left\|M_{i j 2}\right\|\left\|N_{i j 2}\right\|\right)\left\|z_{j 2}\right\|, \\
& \sum_{i=1}^{L} \sum_{\substack{j=1 \\
j \neq i}}^{L}\left\|K_{j 2}\right\|\left(\left\|H_{j i 3}\right\|+\left\|M_{j i 2}\right\|\left\|N_{j i 1}\right\|\right)\left\|z_{i 1}\right\| \\
& =\sum_{i=1}^{L} \sum_{\substack{j=1 \\
j \neq i}}^{L}\left\|K_{i 2}\right\|\left(\left\|H_{i j 3}\right\|+\left\|M_{i j 2}\right\|\left\|N_{i j 1}\right\|\right)\left\|z_{j 1}\right\|,
\end{aligned}
$$

the above inequality can be rewritten as

$$
\begin{aligned}
\dot{V} \leq \sum_{i=1}^{L} & {\left[\left\|K_{i 2}\right\|\left(\left\|A_{i 3}\right\|+\left\|D_{i 2}\right\|\left\|E_{i 1}\right\|\right)\left\|z_{i 1}\right\|\right.} \\
& +\left\|K_{i 2}\right\|\left(\left\|A_{i 4}\right\|+\left\|D_{i 2}\right\|\left\|E_{i 2}\right\|\right)\left\|z_{i 2}\right\| \\
& \left.+\beta_{i}\left\|K_{i} C_{i 2}^{-1}\right\|\left\|y_{i}(0)\right\| \exp \left(-\beta_{i} t\right)\right] \\
+ & \sum_{i=1}^{L} \sum_{\substack{j=1 \\
j \neq i}}^{L}\left\|K_{j 2}\right\|\left(\left\|H_{j i 4}\right\|+\left\|M_{j i 2}\right\|\left\|N_{j i 2}\right\|\right)\left\|z_{i 2}\right\| \\
+ & \sum_{i=1}^{L} \sum_{\substack{j=1 \\
j \neq i}}^{L}\left\|K_{j 2}\right\|\left(\left\|H_{j i 3}\right\|+\left\|M_{j i 2}\right\|\left\|N_{j i 1}\right\|\right)\left\|z_{i 1}\right\| \\
+ & \sum_{i=1}^{L} \frac{\sigma_{i}^{T}}{\left\|\sigma_{i}\right\|} K_{i 2} B_{i 2} u_{i}+\sum_{i=1}^{L}\left\|K_{i 2}\right\|\left\|B_{i 2}\right\|\left\|\xi_{i}\left(x_{i}, t\right)\right\| .
\end{aligned}
$$

In addition, $x_{i}=T_{i}^{-1} z_{i}$, where $T_{i}^{-1}=\left[\begin{array}{ll}W_{i 1} & W_{i 2}\end{array}\right], z_{i}=\left[\begin{array}{l}z_{i 1} \\ z_{i 2}\end{array}\right]$ and using Assumption 6, we have

$$
\begin{aligned}
\left\|\xi_{i}\left(x_{i}, t\right)\right\| & \leq c_{i}+b_{i}\left\|x_{i}(t)\right\| \\
& =c_{i}+b_{i}\left(\left\|W_{i 1}\right\|\left\|z_{i 1}\right\|+\left\|W_{i 2}\right\|\left\|z_{i 2}\right\|\right) .
\end{aligned}
$$

Substituting (54) into (53), we obtain

$$
\begin{aligned}
\dot{V} \leq \sum_{i=1}^{L}\left[\left\|K_{i 2}\right\|\left(\left\|A_{i 3}\right\|+\left\|D_{i 2}\right\|\left\|E_{i 1}\right\|\right)\left\|z_{i 1}\right\|\right. \\
+\left\|K_{i 2}\right\|\left(\left\|A_{i 4}\right\|+\left\|D_{i 2}\right\|\left\|E_{i 2}\right\|\right)\left\|z_{i 2}\right\| \\
+\sum_{i=1}^{L} \frac{\sigma_{i}^{T}}{\left\|\sigma_{i}\right\|} K_{i 2} B_{i 2} u_{i} \\
\left.+\beta_{i}\left\|K_{i} C_{i 2}^{-1}\right\|\left\|y_{i}(0)\right\| \exp \left(-\beta_{i} t\right)\right] \\
+\sum_{\substack{i=1 \\
\sum_{j=1}^{L}}}^{L}\left\|K_{j 2}\right\|\left(\left\|H_{j i 4}\right\|+\left\|M_{j i 2}\right\|\left\|N_{j i 2}\right\|\right)\left\|z_{i 2}\right\| \\
+\sum_{\substack{j \neq i \\
\sum_{i=1}^{L}}}^{L}\left\|K_{j 2}\right\|\left(\left\|H_{j i 3}\right\|+\left\|M_{j i 2}\right\|\left\|N_{j i 1}\right\|\right)\left\|z_{i 1}\right\|
\end{aligned}
$$

$$
+\sum_{i=1}^{L}\left\|K_{i 2}\right\|\left\|B_{i 2}\right\|\left[c_{i}+b_{i}\left(\left\|W_{i 1}\right\|\left\|z_{i 1}\right\|+\left\|W_{i 2}\right\|\left\|z_{i 2}\right\|\right)\right] .
$$

Equation (8) implies that

$$
\left\|z_{i 2}\right\| \leq\left\|K_{i 2}^{-1}\right\|\left\|K_{i} C_{i 2}^{-1}\right\|\left\|y_{i}\right\|
$$

Now, for the design of a controller using only output variables, we apply Lemma 17 to the system (4). Let $v_{i 1}=z_{i 1}, v_{i 2}=z_{i 2}$, $S_{i 1}=A_{i 1}, G_{i 1}=D_{i 1}, G_{i 2}=D_{i 2}, X_{i 1}=E_{i 1}, X_{i 2}=E_{i 2}, \Delta_{i}=F_{i}$, $S_{i 2}=A_{i 2}, \Delta_{i j}=F_{i j}, A_{i j 1}=H_{i j 1}, A_{i j 2}=H_{i j 2}, D_{i j 1}=M_{i j 1}$, $D_{i j 2}=M_{i j 2}, E_{i j 1}=N_{i j 1}$, and $E_{i j 2}=N_{i j 2}$. Then, from (32), (46), and (56), we obtain

$$
\begin{gathered}
\phi_{i}(t)=\eta_{i}(t), \\
\sum_{i=1}^{L}\left\|z_{i 1}\right\| \leq \sum_{i=1}^{L} \eta_{i}(t) .
\end{gathered}
$$

Using (56) and (58), the inequality (55) can be rewritten as

$$
\begin{aligned}
\dot{V} \leq \sum_{i=1}^{L}[ & \left\|K_{i 2}\right\|\left(\left\|A_{i 3}\right\|+\left\|D_{i 2}\right\|\left\|E_{i 1}\right\|\right) \eta_{i} \\
& +\left\|K_{i 2}\right\|\left(\left\|A_{i 4}\right\|+\left\|D_{i 2}\right\|\left\|E_{i 2}\right\|\right) \\
& \cdot\left\|K_{i 2}^{-1}\right\|\left\|K_{i} C_{i 2}^{-1}\right\|\left\|y_{i}\right\| \\
& \left.+\beta_{i}\left\|K_{i} C_{i 2}^{-1}\right\|\left\|y_{i}(0)\right\| \exp \left(-\beta_{i} t\right)\right]
\end{aligned}
$$




$$
\begin{aligned}
& +\sum_{\substack{j=1 \\
j \neq 1 \\
j \neq i}}^{L}\left\|K_{j 2}\right\|\left(\left\|H_{j i 3}\right\|+\left\|M_{j i 2}\right\|\left\|N_{j i 1}\right\|\right) \eta_{i} \\
& +\sum_{i=1}^{L} \sum_{\substack{j=1 \\
j \neq i}}^{L}\left\|K_{j 2}\right\|\left(\left\|H_{j i 4}\right\|+\left\|M_{j i 2}\right\|\left\|N_{j i 2}\right\|\right) \\
& +\sum_{i=1}^{L} \frac{\sigma_{i}^{T}}{\left\|\sigma_{i}\right\|} K_{i 2} B_{i 2} u_{i} \\
& +\sum_{i=1}^{L} b_{i}\left\|K_{i 2}\right\|\left\|B_{i 2}\right\|\left(\left\|W_{i 1}\right\| \eta_{i}+\left\|W_{i 2}\right\|\right. \\
& +\sum_{i=1}^{L} c_{i}\left\|K_{i 2}\right\|\left\|C_{i 2}\right\| y_{i} \| \\
& \left..\left\|K_{i 2}^{-1}\right\|\left\|K_{i} C_{i 2}^{-1}\right\|\left\|y_{i}\right\|\right)
\end{aligned}
$$

Inserting the control law (44) into the right-hand side of (59) yields

$$
\begin{aligned}
& \dot{V} \leq \sum_{i=1}^{L}\left[\left\|K_{i 2}\right\|\left(\left\|A_{i 3}\right\|+\left\|D_{i 2}\right\|\left\|E_{i 1}\right\|\right) \eta_{i}\right. \\
& +\left\|K_{i 2}\right\|\left(\left\|A_{i 4}\right\|+\left\|D_{i 2}\right\|\left\|E_{i 2}\right\|\right)\left\|K_{i 2}^{-1}\right\|\left\|K_{i} C_{i 2}^{-1}\right\|\left\|y_{i}\right\| \\
& \left.+\beta_{i}\left\|K_{i} C_{i 2}^{-1}\right\|\left\|y_{i}(0)\right\| \exp \left(-\beta_{i} t\right)\right] \\
& +\sum_{i=1}^{L} \sum_{\substack{j=1 \\
j \neq i}}^{L}\left\|K_{j 2}\right\|\left(\left\|H_{j i 3}\right\|+\left\|M_{j i 2}\right\|\left\|N_{j i 1}\right\|\right) \eta_{i} \\
& +\sum_{i=1}^{L} \sum_{\substack{j=1 \\
j \neq i}}^{L}\left\|K_{j 2}\right\|\left(\left\|H_{j i 4}\right\|+\left\|M_{j i 2}\right\|\left\|N_{j i 2}\right\|\right) \\
& \cdot\left\|K_{i 2}^{-1}\right\|\left\|K_{i} C_{i 2}^{-1}\right\|\left\|y_{i}\right\| \\
& +\sum_{i=1}^{L} b_{i}\left\|K_{i 2}\right\|\left\|B_{i 2}\right\|\left(\left\|W_{i 1}\right\| \eta_{i}+\left\|W_{i 2}\right\|\right. \\
& \left.\cdot\left\|K_{i 2}^{-1}\right\|\left\|K_{i} C_{i 2}^{-1}\right\|\left\|y_{i}\right\|\right) \\
& +\sum_{i=1}^{L} c_{i}\left\|K_{i 2}\right\|\left\|B_{i 2}\right\| \\
& -\sum_{i=1}^{L} \frac{\sigma_{i}^{T}}{\left\|\sigma_{i}\right\|} K_{i 2} B_{i 2}\left(K_{i 2} B_{i 2}\right)^{-1}\left(\widehat{\kappa}_{i}+\kappa_{i}\left\|y_{i}\right\|+\bar{\kappa}_{i} \eta_{i}\right) \frac{\sigma_{i}}{\left\|\sigma_{i}\right\|} .
\end{aligned}
$$

Therefore,

$$
\dot{V} \leq-\sum_{i=1}^{L} \alpha_{i}<0 .
$$

It is then clear that $\dot{V}\left(\sigma_{i}\left(x_{i}(t), t\right)\right)<0$. Since $\sigma_{i}\left(x_{i}(0), 0\right)=0$, suppose that there exists a time $t>0$ such that

$$
\begin{aligned}
V\left(\sigma_{i}\left(x_{i}(t), t\right)\right) & =\sum_{i=1}^{L}\left\|\sigma_{i}\left(x_{i}(t), t\right)\right\| \leq V\left(\sigma_{i}\left(x_{i}(0), 0\right)\right) \\
& =\sum_{i=1}^{L}\left\|\sigma_{i}\left(x_{i}(0), 0\right)\right\|=0 .
\end{aligned}
$$

Thus, the identities

$$
\sigma_{i}\left(x_{i}(t), t\right)=\dot{\sigma}_{i}\left(x_{i}(t), t\right)=0
$$

hold for all $t \geq 0$; that is, there is no reaching phase and the system states remain on the sliding mode for all time $t \geq 0$. Thus, the proof is completed.

Remark 20. From sliding mode control theory, Theorems 14 and 19 together show that the sliding surface (7) with the decentralized output feedback control law (44) guarantees the following: (1) at any initial value, the system states remain on the sliding surface for all time $t \geq 0$ and (2) the complex interconnected system (1) in the sliding mode is asymptotically stable.

Remark 21. Unlike the existing related work such as [13-25], the stability of interconnected system (1) can be assured for all time.

Remark 22. In contrast to other SMC approaches such as those presented in [1,7-12], the proposed method can be applied to complex interconnected systems where only output information is available.

Remark 23. It is obvious that this approach uses the output information completely in the sliding surface and controller design. Therefore, conservatism is reduced and robustness is enhanced.

\section{Numerical Examples}

To verify the effectiveness of the proposed decentralized output feedback SMC law, we apply our single-phase SMC to a mismatched uncertain interconnected system composed of 
two third-order subsystems, which is modified from [30] as follows:

$$
\begin{aligned}
\dot{x}_{1}= & \left(\left[\begin{array}{ccc}
-8 & 0 & 1 \\
0 & -7 & 1 \\
1 & 0 & 0
\end{array}\right]+\Delta A_{1}\right) x_{1}+\left[\begin{array}{l}
0 \\
0 \\
1
\end{array}\right]\left(u_{1}+\xi_{1}\left(x_{1}, t\right)\right) \\
& +\left(\left[\begin{array}{lll}
1 & 0 & 0 \\
0 & 0 & 1 \\
0 & 1 & 0
\end{array}\right]+\Delta H_{12}\right) x_{2}, \\
\dot{x}_{2}= & \left(\left[\begin{array}{ccc}
-6 & 0 & 1 \\
0 & -7 & 1 \\
1 & 0 & 0
\end{array}\right]+\Delta A_{2}\right) x_{2}+\left[\begin{array}{l}
0 \\
0 \\
1
\end{array}\right]\left(u_{2}+\xi_{2}\left(x_{2}, t\right)\right) \\
& +\left(\left[\begin{array}{lll}
1 & 0 & 0 \\
0 & 1 & 0 \\
0 & 1 & 0
\end{array}\right]+\Delta H_{21}\right) x_{1}, \\
y_{i}= & {\left[\begin{array}{lll}
1 & 1 & 0 \\
0 & 0 & 1
\end{array}\right] x_{i}, \quad i=1,2 }
\end{aligned}
$$

where $x_{1}=\left[\begin{array}{l}x_{11} \\ x_{12} \\ x_{13}\end{array}\right] \in R^{3}, x_{2}=\left[\begin{array}{l}x_{21} \\ x_{22} \\ x_{23}\end{array}\right] \in R^{3}, u_{1} \in R^{1}$, $y_{1}=\left[\begin{array}{l}y_{11} \\ y_{12}\end{array}\right] \in R^{2}, u_{2} \in R^{1}$, and $y_{2}=\left[\begin{array}{l}y_{21} \\ y_{22}\end{array}\right] \in R^{2}$. The mismatched uncertainties in the state matrix are assumed to satisfy $\Delta A_{1}=\left[\begin{array}{lll}0.1 & 0.1 & 0\end{array}\right]^{T} F_{1}\left[\begin{array}{lll}0.1 & 0 & 0\end{array}\right]$ and $\Delta A_{2}=$ $\left[\begin{array}{lll}0 & 0.1 & 0.1\end{array}\right]^{T} F_{2}\left[\begin{array}{lll}0 & 0 & 1\end{array}\right]$ with

$$
\begin{aligned}
& F_{1}=0.9 \sin \left(x_{11}^{2} x_{13}+t \times x_{12}+x_{13}+t \times x_{11} x_{12}\right), \\
& F_{2}=0.9 \sin \left(x_{21} x_{23}+x_{23}^{2} x_{22}+t \times x_{22}+x_{21} x_{22}\right) .
\end{aligned}
$$

The mismatched interconnections are given by $\Delta H_{12}=\left[\begin{array}{lll}0.1 & 0 & 0.1\end{array}\right]^{T} F_{12}\left[\begin{array}{lll}0.1 & 0 & 0.1\end{array}\right]$ and $\Delta H_{21}=$ $\left[\begin{array}{lll}0.1 & 0.1 & 0.1\end{array}\right]^{T} F_{21}\left[\begin{array}{lll}0 & 0.1 & 0.1\end{array}\right]$ with

$$
\begin{aligned}
& F_{12}=0.8 \sin \left(x_{22} x_{23}+x_{22}+t \times x_{21} x_{22}\right), \\
& F_{21}=0.7 \sin \left(x_{11} x_{13}+t \times x_{12}+x_{11} x_{12} x_{13}\right) .
\end{aligned}
$$

The exogenous disturbances are given as follows: $\left.\| \xi_{1}\left(x_{1}, t\right)\right)\|\leq 1.2+1.3\| x_{1} \|$ and $\left.\| \xi_{2}\left(x_{2}, t\right)\right)\|\leq 2+2.1\| x_{2} \|$.

For this work, the following parameters are given as follows: $\alpha_{1}=0.04, \alpha_{2}=0.3, \beta_{1}=6.1, \beta_{2}=10.8, \varphi_{1}=0.9$, $\varphi_{2}=0.4, \bar{\varphi}_{1}=0.8, \bar{\varphi}_{2}=0.7, \widehat{\varphi}_{1}=0.5, \widehat{\varphi}_{2}=0.6, \widetilde{\varphi}_{1}=1.1$, $\widetilde{\varphi}_{2}=1.2, \delta_{1}=0.3, \delta_{2}=0.4, \widehat{\delta}_{1}=0.1, \widehat{\delta}_{2}=0.2, \widetilde{\delta}_{1}=0.8$, $\widetilde{\delta}_{2}=0.4, \varepsilon_{1}=\widetilde{\varphi}_{1}^{-1}+\delta_{1}^{-1}+\widetilde{\delta}_{1}^{-1}=5.4924, \varepsilon_{2}=\widetilde{\varphi}_{2}^{-1}+\delta_{2}^{-1}+\widetilde{\delta}_{2}^{-1}=$ 5.8333, $v_{1}=\varphi_{1}^{-1}+\bar{\varphi}_{1}^{-1}=2.3611, v_{2}=\varphi_{2}^{-1}+\bar{\varphi}_{2}^{-1}=3.9286$, $\rho_{1}=\widehat{\varphi}_{1}^{-1}+\widehat{\delta}_{1}^{-1}=12, \rho_{2}=\widehat{\varphi}_{2}^{-1}+\widehat{\delta}_{2}^{-1}=6.6667, k_{1}=1.002$, and $k_{2}=1$. The initial conditions for two subsystems are selected to be $x_{1}(0)=\left[\begin{array}{lll}-11 & 9 & 3.5\end{array}\right]^{T}$ and $x_{2}(0)=\left[\begin{array}{lll}-9 & 10 & -1\end{array}\right]^{T}$, respectively. According to the algorithm given in [30], the coordinate transformations are given by $T_{1}=T_{2}=\left[\begin{array}{lll}1 & 0 & 0 \\ 1 & 1 & 0 \\ 0 & 0 & 1\end{array}\right]$. By solving LMI (14), we find a feasible solution $P_{i}, i=1,2$, as follows: $P_{1}=\left[\begin{array}{cc}0.1361 & -0.0107 \\ -0.0107 & 0.1534\end{array}\right]$ and $P_{2}=\left[\begin{array}{lll}0.2598 & 0.0051 \\ 0.0051 & 0.0673\end{array}\right]$. The matrix $K_{i 2}, i=1,2$, is given as $K_{12}=0.00833$,
$K_{22}=0.0113548$. From (7), the single-phase sliding surface for the systems (64) and (65) is designed as

$$
\begin{aligned}
& \sigma_{1}=\left[\begin{array}{lll}
0 & 0 & 0.0013
\end{array}\right] x_{1}-0.0047 \exp (-6.1 t)=0, \\
& \sigma_{2}=\left[\begin{array}{lll}
0 & 0 & 0.0114
\end{array}\right] x_{2}-0.0114 \exp (-10.8 t)=0 .
\end{aligned}
$$

Then, from Theorem 14, we know that the sliding motion of the systems (64) and (65) associated with the sliding surfaces $\sigma_{1}$ and $\sigma_{2}$ are asymptotically stable. From (44), the decentralized output feedback controller for the systems (64) and (65) are given as

$$
\begin{aligned}
u_{1}(t)=- & 750.2281 \\
\cdot[ & 0.0416+0.0285 \exp (-6.1 t) \\
& \left.+0.0018\left\|y_{1}\right\|+0.0204 \eta_{1}(t)\right] \frac{\sigma_{1}}{\left\|\sigma_{1}\right\|}, \\
u_{2}(t)=- & 88.0679 \\
& \cdot[0.3227+0.1226 \exp (-10.8 t) \\
& \left.+0.025\left\|y_{2}\right\|+0.0518 \eta_{2}(t)\right] \frac{\sigma_{2}}{\left\|\sigma_{2}\right\|},
\end{aligned}
$$

where the time functions $\eta_{1}(t)$ and $\eta_{2}(t)$ are the solution of $\dot{\eta}_{1}(t)=-6.613 \eta_{1}(t)+2.258\left\|y_{1}\right\|$ and $\dot{\eta}_{2}(t)=-5.7 \eta_{2}(t)+$ $3.35\left\|y_{2}\right\|$, respectively.

From Theorem 19, the system states stay on the sliding surface from beginning to end. This is to say that the stability of systems (64) and (65) is guaranteed for all time.

Remark 24. In the example above, the mismatched uncertainties in the state matrix of the systems (64) and (65) are nonlinear and time-variable and the mismatched interconnections are also nonlinear and time-variable, as shown in (67) and (68). Thus, the stability of systems (64) and (65) is more difficult to ensure than that of $[17,30]$. Therefore, the approaches given in $[17,30]$ are not applicable here. From Figures 3 and 4, we can see that the sliding mode exists for all time. Even though the mismatched uncertainties in the state matrix and interconnections of the systems (64) and (65) are nonlinear and time-variable, the systems still exhibit good performance with low control energy, as seen in Figures 1, 2, 5 , and 6.

\section{Conclusion}

In this paper, a single-phase SMC law is presented for decentralized robust stability of complex interconnected systems from the beginning to the end. It is proved that the proposed single-phase SMC guaranteed the robustness of complex interconnected system throughout an entire response of the system starting from the initial time instance. One of the key features of the single-phase SMC scheme is that reaching time, which is required in most of the existing two phases of SMC approaches to stabilize the complex interconnected systems, is removed. As a consequence, the proposed singlephase SMC law can be applied to complex interconnected 


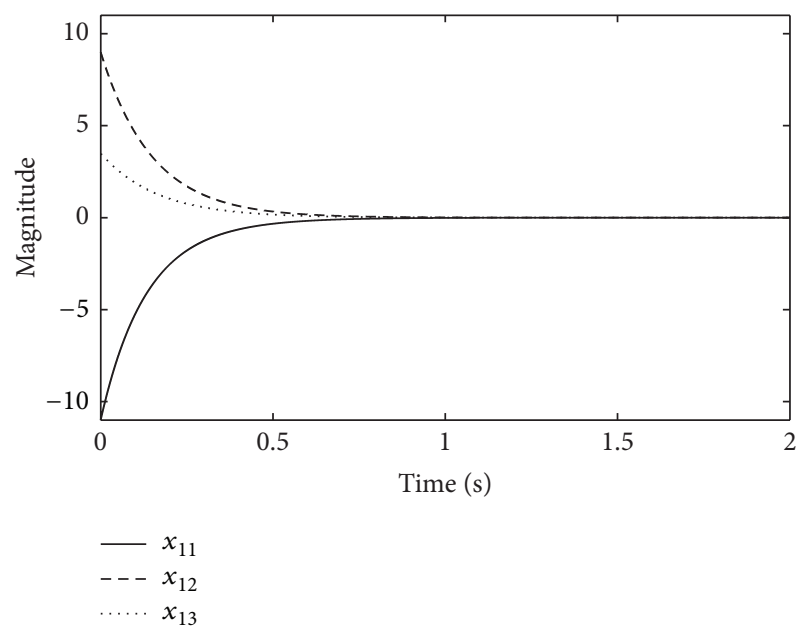

FIGURE 1: Time responses of states $x_{11}$ (solid), $x_{12}$ (dashed), and $x_{13}$ (dotted).

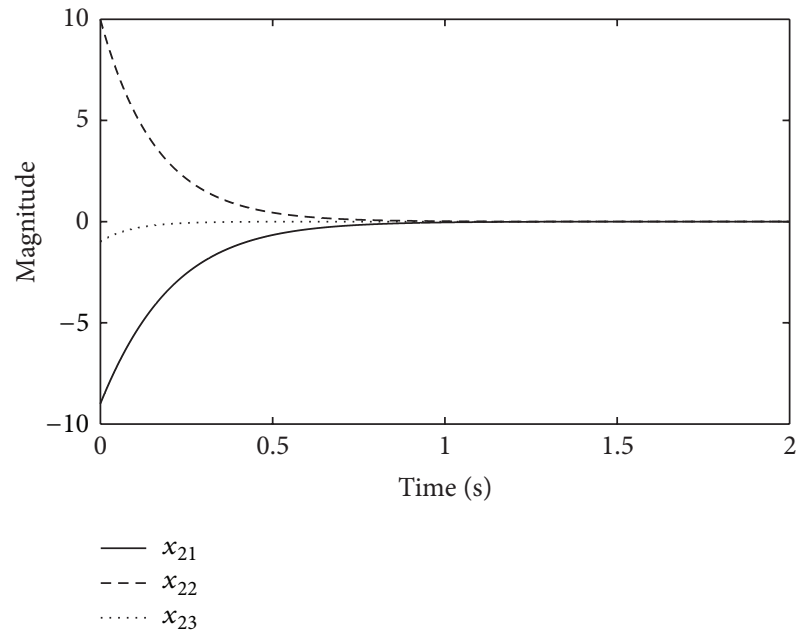

FIgURE 2: Time responses of states $x_{21}$ (solid), $x_{22}$ (dashed), and $x_{23}$ (dotted).

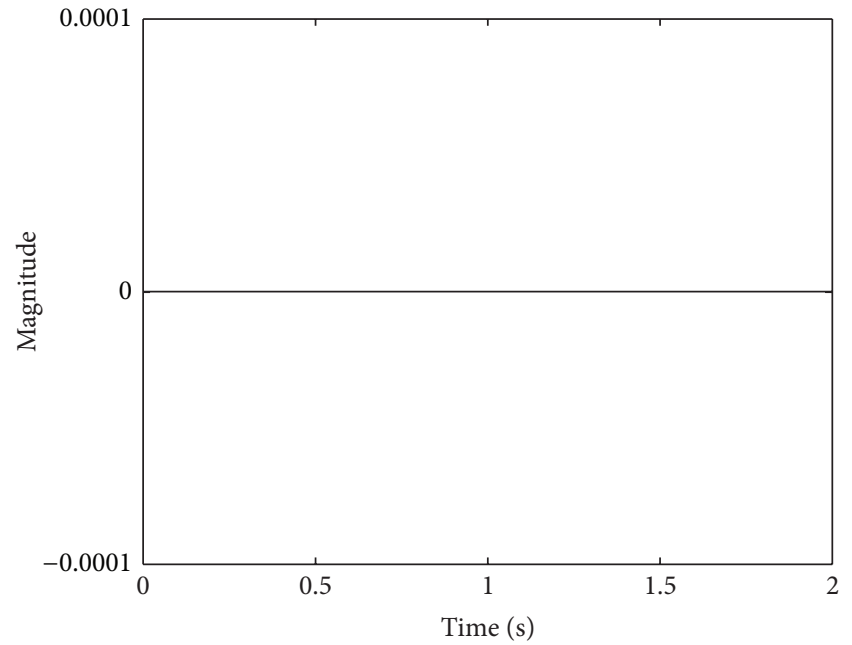

FIGURE 3: Sliding surface $\sigma_{1}$.

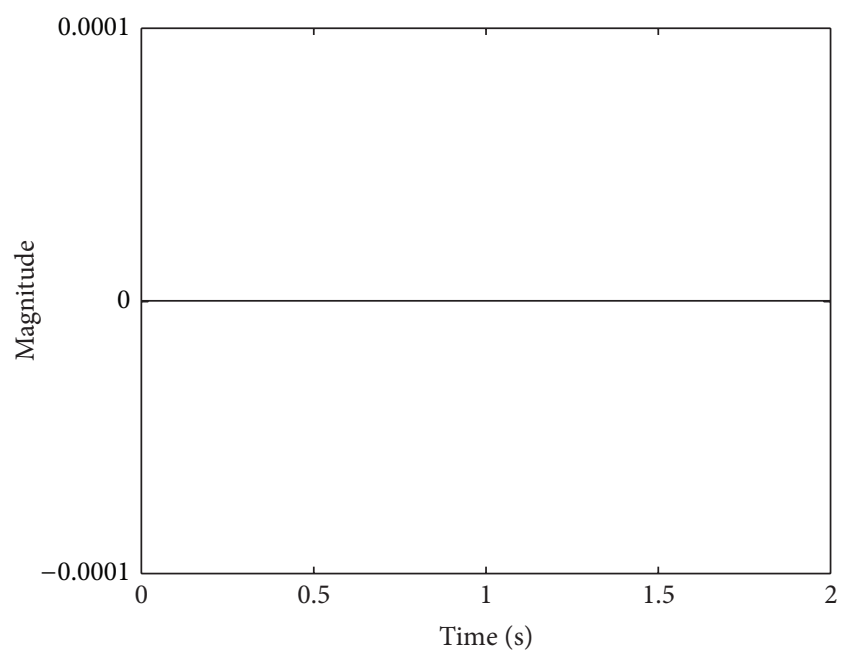

Figure 4: Sliding surface $\sigma_{2}$.

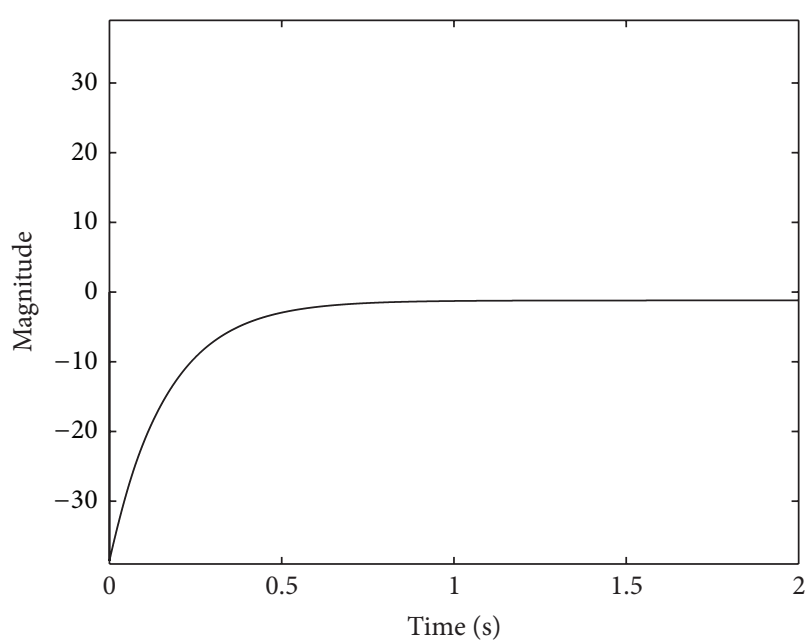

FIGURE 5: Control input $u_{1}$.

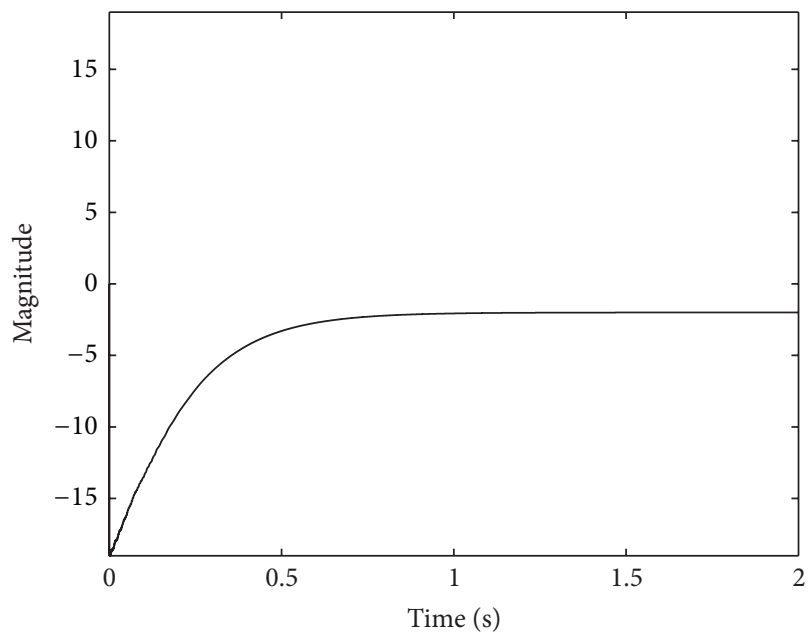

FIGURE 6: Control input $u_{2}$. 
systems, which is not always achievable in the traditional SMC design for complex interconnected systems using only output variables.

\section{Conflict of Interests}

The authors declare that there is no conflict of interests regarding the publication of this paper.

\section{Acknowledgment}

The authors would like to thank the financial support provided by the National Science Council in Taiwan (NSC 1022632-E-212-001-MY3).

\section{References}

[1] H. H. Choi, "LMI-based sliding surface design for integral sliding mode control of mismatched uncertain systems," IEEE Transactions on Automatic Control, vol. 52, no. 4, pp. 736-742, 2007.

[2] H. H. Choi, "An explicit formula of linear sliding surfaces for a class of uncertain dynamic systems with mismatched uncertainties," Automatica, vol. 34, no. 8, pp. 1015-1020, 1998.

[3] S. H. Zak and S. Hui, "On variable structure output feedback controllers for uncertain dynamic systems," IEEE Transactions on Automation Control, vol. 38, no. 10, pp. 1509-1512, 1993.

[4] C. M. Kwan, "On variable structure output feedback controllers," IEEE Transactions on Automatic Control, vol. 41, no. 11, pp. 1691-1693, 1996.

[5] Y.-W. Tsai, K.-H. Mai, and K.-K. Shyu, "Sliding mode control for unmatched uncertain systems with totally invariant property and exponential stability," Journal of the Chinese Institute of Engineers, Transactions of the Chinese Institute of Engineers, Series A, vol. 29, no. 1, pp. 179-183, 2006.

[6] R. J. Mantz, H. de Battista, and P. Puleston, "A new approach to reaching mode of VSS using trajectory planning," Automatica, vol. 37, no. 5, pp. 763-767, 2001.

[7] A. Bartoszewicz and A. Nowacka-Leverton, "SMC without the reaching phase-the switching plane design for the third-order system," IET Control Theory and Applications, vol. 1, no. 5, pp. 1461-1470, 2007.

[8] J. Ackermann and V. Utkin, "Sliding mode control design based on Ackermann's formula," IEEE Transactions on Automatic Control, vol. 43, no. 2, pp. 234-237, 1998.

[9] M. Rubagotti, A. Estrada, F. Castaños, A. Ferrara, and L. Fridman, "Integral sliding mode control for nonlinear systems with matched and unmatched perturbations," IEEE Transactions on Automatic Control, vol. 56, no. 11, pp. 2699-2704, 2011.

[10] Q. Gao, L. Liu, G. Feng, Y. Wang, and J. Qiu, "Universal fuzzy integral sliding-mode controllers based on T-S fuzzy models," IEEE Transactions on Fuzzy Systems, vol. 22, no. 2, pp. 350-362, 2014.

[11] D. Ginoya, P. D. Shendge, and S. B. Phadke, "Sliding mode control for mismatched uncertain systems using an extended disturbance observer," IEEE Transactions on Industrial Electronics, vol. 61, no. 4, pp. 1983-1992, 2014.

[12] M. Liu, L. Zhang, P. Shi, and H. R. Karimi, "Robust control of stochastic systems against bounded disturbances with application to flight control," IEEE Transactions on Industrial Electronics, vol. 61, no. 3, pp. 1504-1515, 2014.
[13] Y.-W. Tsai, K.-K. Shyu, and K.-C. Chang, "Decentralized variable structure control for mismatched uncertain large-scale systems-a new approach," Systems and Control Letters, vol. 43, no. 2, pp. 117-125, 2001.

[14] K. K. Shyu, W. J. Liu, and K. C. Hsu, "Decentralised variable structure control of uncertain large-scale systems containing a dead-zone," IEE Proceedings: Control Theory and Applications, vol. 150, no. 5, pp. 467-475, 2003.

[15] K.-K. Shyu, W.-J. Liu, and K.-C. Hsu, "Design of large-scale time-delayed systems with dead-zone input via variable structure control," Automatica, vol. 41, no. 7, pp. 1239-1246, 2005.

[16] C.-W. Chung and Y. Chang, "Design of a sliding mode controller for decentralised multi-input systems," IET Control Theory \& Applications, vol. 5, no. 1, pp. 221-230, 2011.

[17] K. Kalsi, J. Lian, and S. H. Zak, "Decentralized dynamic output feedback control of nonlinear interconnected systems," IEEE Transactions on Automatic Control, vol. 55, no. 8, pp. 1964-1970, 2010.

[18] X.-G. Yan, S. K. Spurgeon, and C. Edwards, "Global decentralised static output feedback sliding-mode control for interconnected time-delay systems," IET Control Theory and Applications, vol. 6, no. 2, pp. 192-202, 2012.

[19] Z. He, X. Wang, Z. Gao, and J. Bai, "Sliding mode control based on observer for a class of state-delayed switched systems with uncertain perturbation," Mathematical Problems in Engineering, vol. 2013, Article ID 614878, 9 pages, 2013.

[20] W.-J. Wang and J.-L. Lee, "Decentralized variable structure control design in perturbed nonlinear systems," Journal of Dynamic Systems, Measurement and Control, Transactions of the ASME, vol. 115, no. 3, pp. 551-554, 1993.

[21] K.-K. Shyu and J.-J. Yan, "Variable-structure model following adaptive control for systems with time-varying delay," Control Theory and Advanced Technology, vol. 10, no. 3, pp. 513-521, 1994.

[22] K.-C. Hsu, "Decentralized variable-structure control design for uncertain large-scale systems with series nonlinearities," International Journal of Control, vol. 68, no. 6, pp. 1231-1240, 1997.

[23] K.-C. Hsu, "Decentralized variable structure model-following adaptive control for interconnected systems with series nonlinearities," International Journal of Systems Science, vol. 29, no. 4, pp. 365-372, 1998.

[24] C.-H. Chou and C.-C. Cheng, "A decentralized model reference adaptive variable structure controller for large-scale time-varying delay systems," IEEE Transactions on Automatic Control, vol. 48, no. 7, pp. 1213-1217, 2003.

[25] C.-J. Wang and J.-S. Chiou, "On delay independent stabilization analysis for a class of switched large-scale time-delay systems," Abstract and Applied Analysis, vol. 2013, Article ID 849578, 10 pages, 2013.

[26] Y. Cui, K. Liu, Y. Zhao, and X. Wang, "Robust $H_{\infty}$ control for a class of uncertain switched fuzzy time-delay systems based on T-S models," Mathematical Problems in Engineering, vol. 2013, Article ID 234612, 8 pages, 2013.

[27] P. P. Khargonekar, I. R. Petersen, and K. Zhou, "Robust stabilization of uncertain linear systems: quadratic stabilizability and $H^{\infty}$ control theory," IEEE Transactions on Automatic Control, vol. 35, no. 3, pp. 356-361, 1990.

[28] S. Boyd, E. L. Ghaoui, E. Feron, and V. Balakrishna, Linear Matrix Inequalities in System and Control Theory, SIAM, Philadelphia, Pa, USA, 1998. 
[29] D. Cheng, L. Guo, and J. Huang, "On quadratic Lyapunov functions," IEEE Transactions on Automatic Control, vol. 48, no. 5, pp. 885-890, 2003.

[30] X.-G. Yan, C. Edwards, and S. K. Spurgeon, "Decentralised robust sliding mode control for a class of nonlinear interconnected systems by static output feedback," Automatica, vol. 40, no. 4, pp. 613-620, 2004. 


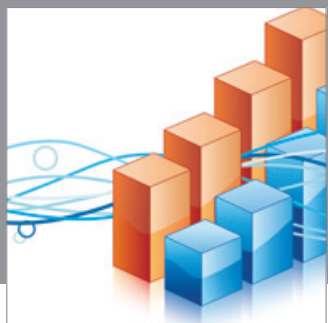

Advances in

Operations Research

mansans

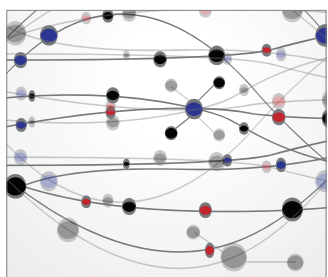

The Scientific World Journal
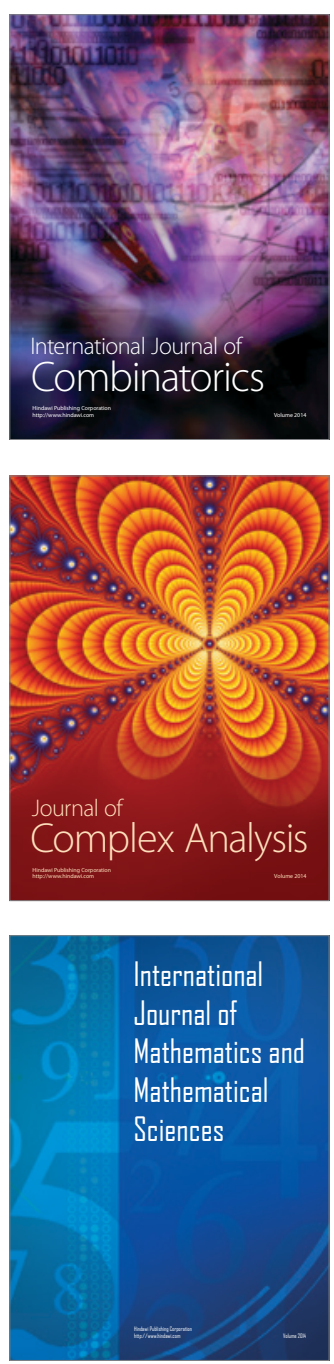
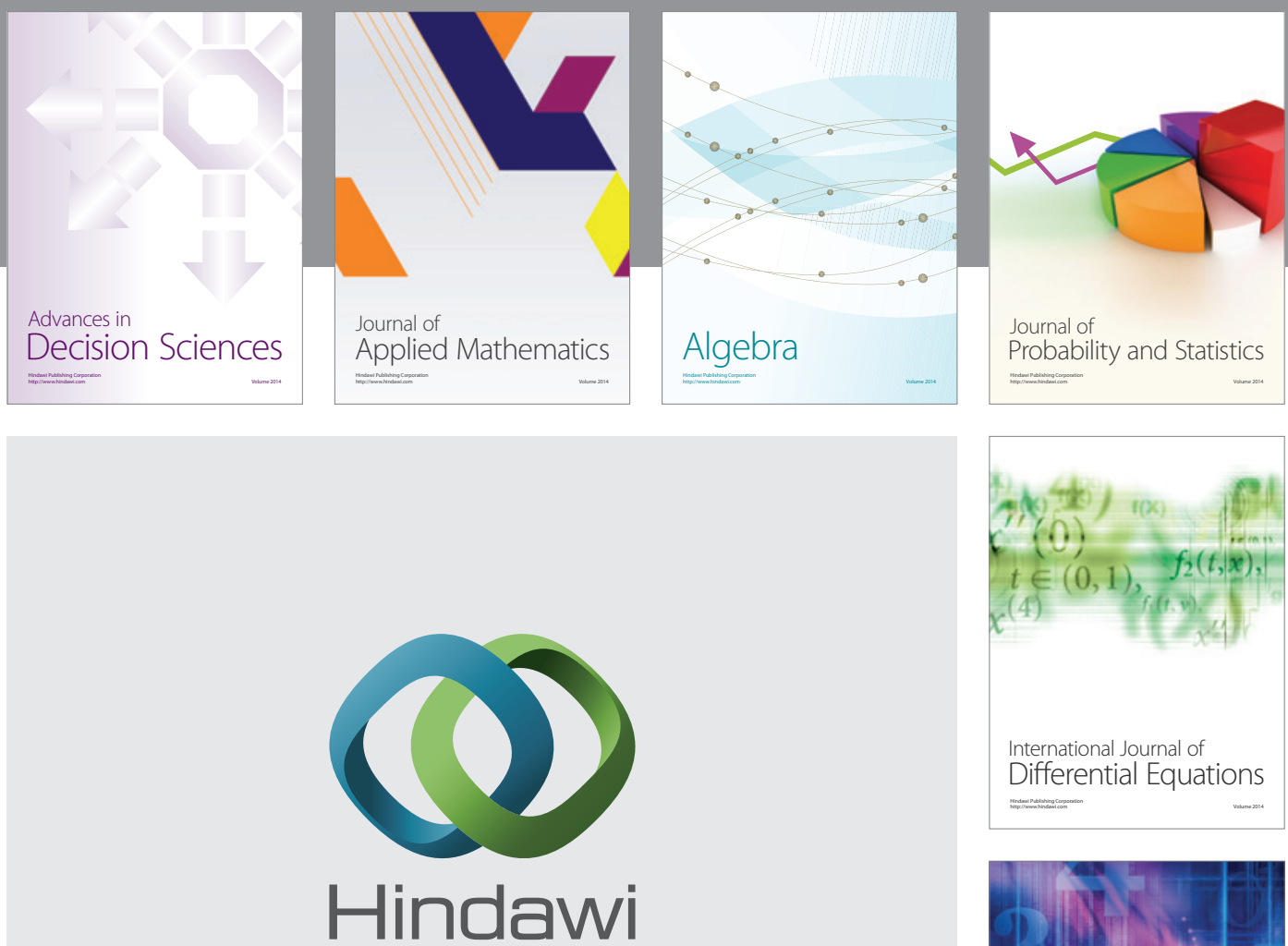

Submit your manuscripts at http://www.hindawi.com
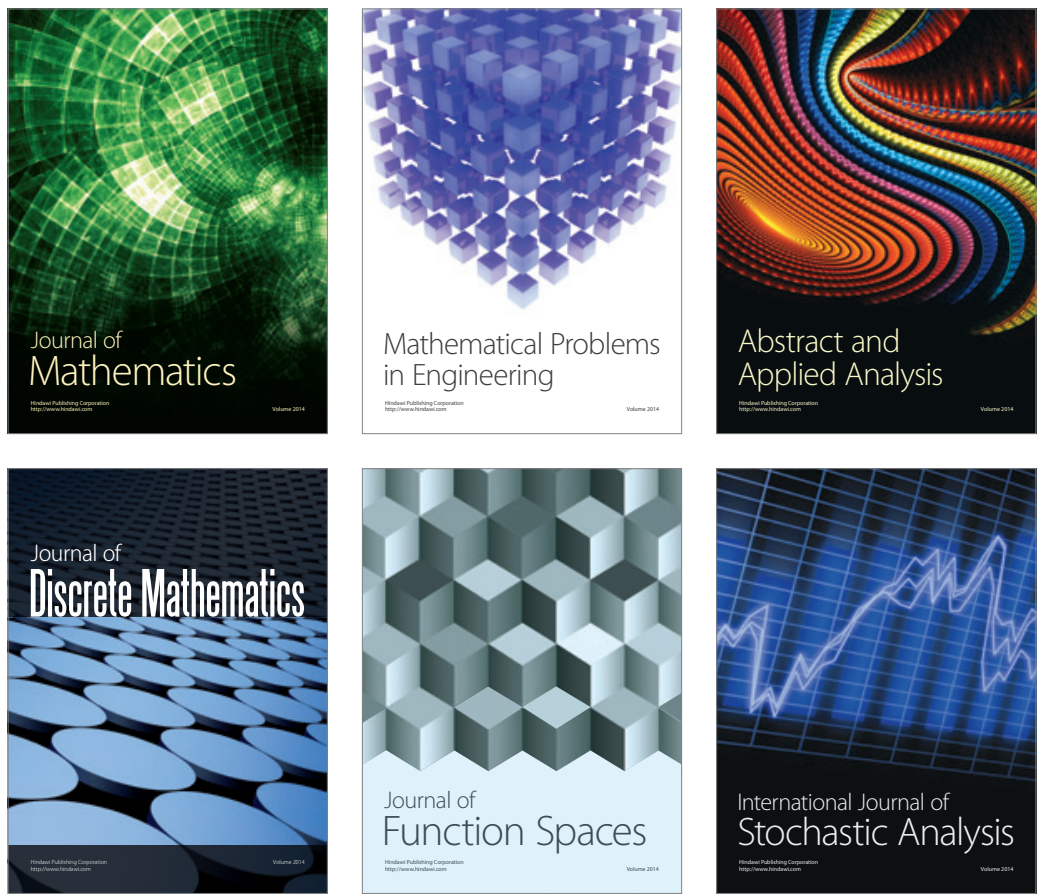

Journal of

Function Spaces

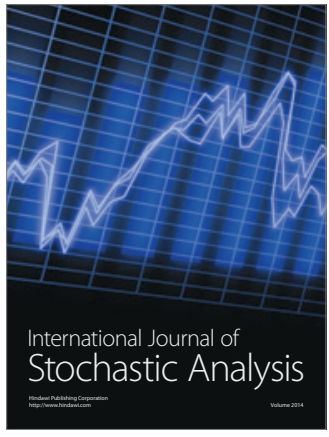

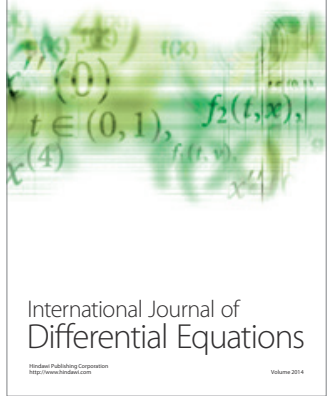
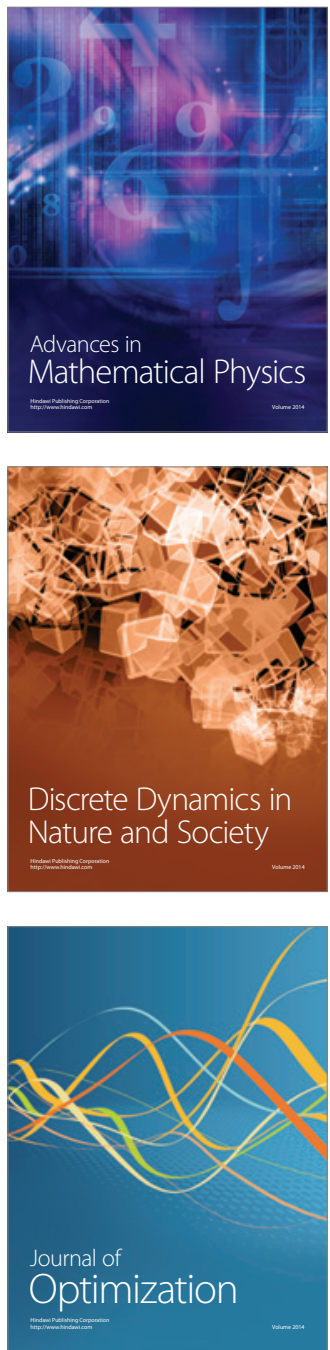